\title{
HGF and CXCL13, Two Antagonizing Cytokines in Lung Inflammation and Fibrosis, Predict the Severity and the Mortality of COVID-19
}

\section{Matthieu Perreau}

Universitaire Vaudois (CHUV)

Madeleine Suffiotti

Lausanne University Hospital

\section{Pedro Marques-Vidal}

University Hospital of Lausanne https://orcid.org/0000-0002-4548-8500

\section{Aurelie Wiedemann}

Vaccine Research Institute, Université Paris-Est Créteil, Faculté de Médecine, INSERM U955, Team 16,

Créteil, France. https://orcid.org/0000-0002-4224-3137

\section{Yves Levy}

Vaccine Research Institute https://orcid.org/0000-0002-5549-6256

\section{Cédric Laouénan}

AP-HP, Hôpital Bichat, Département Épidémiologie Biostatistiques et Recherche Clinique, INSERM, Centre d'Investigation clinique-Epidémiologie Clinique

\section{Jade Ghosn}

AP-HP, Hôpital Bichat, Service de Maladies Infectieuses et Tropicales,

\section{Craig Fenwick}

Service of Immunology and Allergy, Lausanne University Hospital, University of Lausanne

\section{Denis Comte}

Lausanne University Hospital

\section{Thierry Roger}

Lausanne University Hospital and University of Lausanne https://orcid.org/0000-0002-9358-0109

\section{Jean Regina}

Lausanne University Hospital https://orcid.org/0000-0003-2219-9834

\section{Peter Vollenweider}

Lausanne University Hospital (CHUV)

\section{Gerard Waeber}

CHUV University Hospital https://orcid.org/0000-0003-4193-788X

\section{Mauro Oddo}

Lausanne University Hospital

\section{Thierry Calandra}


Lausanne University Hospital

Giuseppe Pantaleo ( $\square$ Giuseppe.Pantaleo@chuv.ch )

University Hospital of Lausanne

\section{Article}

Keywords: HGF, CXCL13, predictors, severe COVID-19

Posted Date: April 9th, 2021

DOl: https://doi.org/10.21203/rs.3.rs-241266/v1

License: (c) (i) This work is licensed under a Creative Commons Attribution 4.0 International License. Read Full License

Version of Record: A version of this preprint was published at Nature Communications on August 9th, 2021. See the published version at https://doi.org/10.1038/s41467-021-25191-5. 
Keywords: HGF, CXCL13, predictors, severe COVID-19

${ }^{1 \S}$ Matthieu Perreau, ${ }^{1 \S}$ Madeleine Suffiotti, ${ }^{2}$ Pedro Marques-Vidal, ${ }^{3,4}$ Aurelie Wiedemann,

${ }^{3,4}$ Yves Levy, ${ }^{5,6}$ Cédric Laouénan, ${ }^{7}$ Jade Ghosn, ${ }^{¥}$ French COVID cohort study group, ${ }^{1}$ Craig Fenwick, ${ }^{1}$ Denis Comte, ${ }^{8}$ Thierry Roger, ${ }^{8}$ Jean Regina, ${ }^{2}$ Peter Vollenweider, ${ }^{2}$ Gerard Waeber, ${ }^{9}$ Mauro Oddo, ${ }^{8}$ Thierry Calandra, $1,3,10,{ }^{*}$ Giuseppe Pantaleo

${ }^{1}$ Service of Immunology and Allergy, Lausanne University Hospital, University of Lausanne, 1011 Lausanne, Switzerland,

${ }^{2}$ Service of Internal Medicine, Department of Medicine, Lausanne University Hospital, University of Lausanne, 1011 Lausanne, Switzerland

${ }^{3}$ Vaccine Research Institute, Université Paris-Est, Faculté de Médecine, INSERM U955, Créteil, France.

${ }^{4}$ Assistance Publique-Hôpitaux de Paris, Groupe Henri-Mondor Albert-Chenevier, Service d'Immunologie Clinique, Créteil, France

${ }^{5}$ AP-HP, Hôpital Bichat, Département Épidémiologie Biostatistiques et Recherche Clinique, INSERM, Centre d'Investigation clinique-Epidémiologie Clinique 1425 F-75018 Paris, France

${ }^{6}$ Université de Paris, INSERM, IAME UMR 1137, F-75018 Paris, France

${ }^{7}$ AP-HP, Hôpital Bichat, Service de Maladies Infectieuses et Tropicales, F-75018 Paris, France

${ }^{8}$ Service of Infectious Diseases, Lausanne University Hospital, University of Lausanne, 1011 Lausanne, Switzerland

${ }^{9}$ Service of Intensive Care, Lausanne University Hospital, University of Lausanne, 1011 Lausanne, Switzerland

${ }^{10}$ Swiss Vaccine Research Institute, Lausanne University Hospital, University of Lausanne, 1011 Lausanne, Switzerland

$\S$ Authors contributed equally

${ }^{¥}$ Members of the French COVID study group are in listed in Supplementary Information

* Correspondence should be addressed to:

Prof. Giuseppe Pantaleo (Giuseppe.Pantaleo@chuv.ch)

Service d'immunologie et allergie, Rue du Bugnon 46, Lausanne, Switzerland

Running title: Immunological Predictors of severe COVID-19

Abstract word count: 184 
39 Abstract. The objective of the present study was to identify biological signatures of severe 40 COVID-19 predictive of admission in the intensive care unit (ICU). Over 170 immunological 41 markers were investigated in a 'discovery' cohort ( $\mathrm{n}=98$ patients) of the Lausanne University 42 Hospital (LUH-1). While cellular immunological markers lacked power in discriminating 43 between ICU and non-ICU patients, 13 out of 49 cytokines were significantly associated with 44 ICU admission in the three cohorts $(P<0.05$ to $P<0.001)$. The cytokine results were confirmed 45 in two 'validation' cohorts, i.e. the French COVID-19 Study (FCS; n=62) and a second LUH462 cohort $(n=47)$. Of note, HGF is a pleiotropic cytokine with anti-inflammatory properties 47 playing a fundamental role in lung tissue repair, and CXCL13, a pro-inflammatory chemokine associated with pulmonary fibrosis and regulating the maturation of B cell response. The two cytokines in combination were the best predictors of ICU admission (positive and negative 50 predictive values ranging from $81.8 \%$ to $93.1 \%$ and $85.2 \%$ to $94.4 \%$ in the 3 cohorts) and 51 occurrence of death during patient follow-up ( 8.8 fold higher likelihood of death when both 52 cytokines were increased). Up-regulation of HGF reflects the most powerful counter-regulatory 53 mechanism of the host immune response to antagonize the pro-inflammatory cytokines including CXCL13 and to prevent lung fibrosis in COVID-19 patients. 


\section{Introduction}

Severe acute respiratory syndrome coronavirus 2 (SARS-CoV-2), the cause of coronavirus disease 19 (COVID-19) induces a broad range of clinical manifestations including asymptomatic infection, mild disease and a life-threatening severe clinical syndrome characterized by respiratory failure, shock and multi-organ dysfunction requiring admission in the intensive care unit (ICU). The severe COVID-19 is associated with a mortality of 5\% to $10 \%$

Several studies have hypothesized that the severity of COVID-19 results from an excessive inflammatory immune response that may cause a life-threatening multi-organ systemic clinical syndrome ${ }^{4-6}$. Similar to SARS-CoV, the inflammatory innate response is mainly due to a massive cytokine and chemokine release syndrome $e^{7,8}$. Patients with COVID19 have elevated serum levels of cytokines (interleukin-1 (IL-1), IL-6, IL-2, IL-7, IL-10, IL12, and IFN- $\gamma$,), chemokines (CCL2, CCL3, CXCL8, CXCL9, and CXCL10, CXCL11) and growth factors (G-CSF and HGF $)^{9,10}$. Recently, Marie Del Valle et al. showed in a retrospective analysis that high serum levels of IL-6, IL- 8 and TNF- $\alpha$ at the time of hospitalization were strong and independent predictors of patient survival ${ }^{11}$. Additional studies performed on a limited number of patients $(n=<50)$, proposed that in addition to cytokines and chemokines, a neutrophil activation signature, monocyte chemoattractants, pro-apoptotic factors and HGF were associated with severe COVID-19 ${ }^{12,13}$. Based on these studies, it is likely that the cytokine release syndrome may drive immune cell infiltration, lung epithelial and endothelial cells apoptosis $^{14}$, suboptimal T-cell function ${ }^{15}$, multi-organ failure, and ultimately death ${ }^{16}$.

Lymphocytopenia is also a hallmark of SARS-CoV2 infection and correlates with disease severity and death ${ }^{17,18}$. Indeed, patients with severe COVID-19 harbor a marked decrease in the absolute cell counts of T cells (both CD4 and CD8), B cells and NK cells ${ }^{18}$. However, a number of studies have shown robust CD8 and/or CD4 $\mathrm{T}$ cell activation and proliferation compared to healthy controls in the majority of patients studied ${ }^{19-21}$. Virus-specific CD4 and CD8 $\mathrm{T}$ cell responses were predominantly directed against the $\mathrm{M}$, Spike, and $\mathrm{N}$ proteins ${ }^{21}$, tended to have a central memory phenotype $\left(\mathrm{CD} 27^{+} \mathrm{CD} 45 \mathrm{RO}^{+}\right)$and consisted of both mono and/or polyfunctional CD4 (IFN $\gamma$, IL-2, and TNF) and CD8 (IFN- $\gamma$, TNF, CD107a) T cells ${ }^{22}$. However, there was no correlation between the function of virus-specific CD4 and CD8 T cells and disease severity ${ }^{22}$.

Recent studies have indicated that an immunodeficient type I interferon (both IFN- $\alpha$ and $\beta$ ) response is associated with excessive inflammation and severe disease and about $10 \%$ of 
patients with anti-IFN antibodies experience severe disease requiring hospitalization in ICU. Add the references of the Science articles.

In the present study, we investigated over 170 immunological parameters to identify signatures associated with the severity of COVID-19 at hospital admission. We studied three cohorts, one 'discovery' (LUH-1) and two 'validation' cohorts (FCS and LUH-2) including a total of 207 patients of which 85 were ICU patients and 122 non-ICU patients. We have identified two cytokines, i.e. $\mathrm{HGF}$ and $\mathrm{CXCL13}$, as the best immunological signature predicting the severity of COVID-19 requiring ICU admission.

\section{Results}

\section{Patient cohorts}

The aim of the present study was to define the immune inflammatory profile of SARSCoV-2 infection and to determine whether unique immune signatures may help identify patients with severe COVID-19 requiring ICU admission, referred to as ICU patients, versus those with moderate COVID-19 admitted in the internal medicine ward, referred to as non-ICU patients. To achieve this objective, 98 adult patients with a PCR-confirmed SARS-CoV2 infection sequentially admitted to the Lausanne University Hospital were enrolled in a 'discovery' cohort (LUH-1) between March $12^{\text {th }}$ and April the $4^{\text {th }}$. Amongst the 98 patients, 43 were admitted directly to the ICU and 55 to the internal medicine ward. Blood and serum samples were collected at the time of admission and ex vivo cellular and serum immune signatures were determined using mass cytometry and multiplex beads assay. After the identification of immune signatures differentiating ICU from non-ICU patients in the 'discovery' cohort, the unique signatures were confirmed in 62 patients enrolled in the FCS cohort including 31 ICU and 31 non-ICU patients, and additional 47 patients in the LUH-2 cohort including 11 ICU and 36 non-ICU patients. The patients of the FCS and LUH-2 validation cohorts were enrolled between January $25^{\text {th }} 2020$ and April the $8^{\text {th }} 2020$ and April $7^{\text {th }}$ and October $15^{\text {th }}$, respectively, and the immunological profiles were analyzed blindly. Reference values for the immunological parameters investigated were derived from the analyses of a separate cohort of 450 healthy donors balanced for gender and age.

Demographic and clinical data of the patients enrolled in the 'discovery' cohort are summarized in Supplemental Table 1. Admission to the ICU for the LUH-1 followed the recommendations of the guidelines of the Swiss Federal Office of Public Health. This may explain the lack of difference for certain demographic parameters such as age and comorbidities between ICU and non-ICU patients. 
The most common symptoms included fever, cough, dyspnea, fatigue, myalgia/arthralgia, nausea/vomiting and anosmia/dysgueusia (Supplemental Table 1). No significant differences in comorbidities were observed between non-ICU and ICU patients $(P>0.05)$. Complications were more frequently observed in ICU than in non-ICU patients $(P<0.05)$ including acute respiratory distress syndrome, community-acquired or hospitalacquired pneumonia, pulmonary embolism, septic shock and acute hepatic injury (Supplemental Table 2).

The oxygen saturation was significantly lower in ICU patients than in non-ICU patients (95\% versus $97 \% ; P<0.05$ ), while the $\mathrm{FIO}_{2}$ was significantly higher in ICU than in non-ICU patients (43\% versus $21 \%$; $P<0.05$ ) (Supplemental Table 1$)$. Total white cell blood count was significantly higher in ICU than in non-ICU patients $\left(8.3\right.$ versus $6.7 \times 10^{9} /$ Liter; $P<0.05$ ) (Supplemental Table 1). Consistent with other studies ${ }^{23}$, clinical parameters of inflammation such as $\mathrm{C}$ reactive protein $(\mathrm{CRP})$, pro-calcitonin and ferritin were markedly elevated and significantly higher in ICU than in non-ICU patients $(P<0.003)$ (Supplemental Table 1).

Finally, ICU patients were more frequently treated with tocilizumab, any antibiotic therapy, inhibitors of the renin-angiotensin-aldosterone system than non-ICU patients $(P<0.001)$ (Supplemental Table 3).

\section{Immune profile of circulating cell populations in ICU and non-ICU patients}

To determine the immune profile of ICU and non-ICU patients we investigated over 170 immunological parameters. We first assessed the influence of SARS-CoV2 infection on the absolute blood counts of CD4 and CD8 T-, B-, gamma-delta T-, NK, monocytic and dendritic cell populations using a panel of 45 surface markers by mass cytometry. Blood samples were collected from the $38 \mathrm{ICU}$ and 53 non-ICU individuals enrolled in the 'discovery' cohort and compared to the reference normal value of 63 blood samples of healthy donors. ICU and nonICU patients showed significant $\mathrm{T}$ cell lymphocytopenia $(P<0.05)$ (Supplemental Figure 1$)$. With regard to CD4 T cells, all CD4 T cell populations were significantly reduced as compared to healthy donors $(P<0.05)$ (Supplemental Figure 1a). CD8 T cells, total, naive and effector memory (EM) cell populations were significantly reduced as compared to healthy donors $(P<0.05)$ while central memory $(\mathrm{CM}) \mathrm{CD} 8 \mathrm{~T}$ cells were significantly increased $(P<0.001)$ and

153 Consistent with a previous study ${ }^{20}$, ICU and non-ICU patients had increased proportion of 154 activated (HLA-DR $\left.{ }^{+} \mathrm{CD} 38^{+}\right)$memory (CD45RA-CD27 $) \mathrm{CD}^{-}$and CD8 $\mathrm{T}$ cells as compared to 155 healthy donors $(P<0.001)$ (Supplemental Figure 1) while no significant differences were 
observed between ICU and non-ICU patients $(P>0.05)$ (Supplemental Figure 1). PD-1 expression increased significantly only on memory (CD45RA-CD27-) CD4 and CD8 T cells in non-ICU patients $(P<0.05)$ (Supplemental Figure 2).

The absolute total B cell number was not substantially influenced by SARS-CoV2 infection $(P>0.05)$ in both ICU and non-ICU patients. However, significant increases were observed in activated B cells (CD21 $\left.1_{\text {low }} \mathrm{CD} 38_{\text {low }}\right)$ and plasma cells $\left(\mathrm{CD} 20_{\text {low }} \mathrm{CD} 38^{\text {high }}\right)$ whereas unswitched memory B cells $\left(\mathrm{CD}^{2} 7^{-} \operatorname{IgD}^{+} \operatorname{IgM}^{+}\right), \operatorname{IgG} 2^{+}$switched memory B cells $\left(\mathrm{CD} 27^{+} \mathrm{IgD}^{-}\right.$ $\left.\operatorname{IgM}^{-} \operatorname{IgG}^{+}\right)$and transitional $\mathrm{B}$ cells $\left(\mathrm{CD} 38^{\text {high }} \mathrm{CD} 24^{\text {high }} \operatorname{IgM}^{+} \operatorname{IgD}^{+} \mathrm{CD} 10^{-}\right)$were significantly reduced as compared to healthy individuals $(P<0.05)$ (Supplemental Figure 1b).

Finally, both ICU and non-ICU patients showed significant reduction in the cell number of gamma-delta T cells, plasmacytoid dendritic cells (DC) (pDC), myeloid, conventional and inflammatory DC populationsas compared to healthy individuals $(P<0.001)$ (Supplemental Figure 1c). Except for gamma-delta T cells, all subsets of innate immune cell populations were more profoundly reduced in ICU patients than in non-ICU patients $(P<0.05$ to $P<0.001)$.

The distribution of different CD4 $\mathrm{T}$ cell lineages and the phosphoprotein signaling profiles were then determined in CD4 T cell populations of 25 ICU and 50 non-ICU patients enrolled in the 'discovery' cohort and compared to blood samples of 146 healthy subjects using two mass cytometry panels composed of 43 and 37 markers. Cumulative data indicated that COVID-19 significantly influenced the distribution of blood CD4 T cell lineages. Indeed, the proportion of T helper type 1 (Th1) $\left(\mathrm{CXCR}^{+} \mathrm{Tbet}^{+}\right)$, Th17 cells $\left(\mathrm{CCR} 6^{+} \mathrm{ROR} \gamma \mathrm{t}^{+}\right)$and Tregs $\left(\mathrm{CD} 25^{+} \mathrm{CD} 127^{-} \mathrm{FoxP}^{+}\right)$were significantly increased at the expense of Th2 cells $\left(\mathrm{CCR} 4^{+} \mathrm{Gata}^{+}\right)$in both ICU and non-ICU patients as compared to healthy individuals $(P<0.001)$ (Figure 1a). However, no significant differences were observed between ICU and non-ICU patients $(P>0.05)$ (Figure 1a).

The ex vivo expression levels of phospho-STAT1 (pSTAT1), pSTAT3 and pSTAT5 were significantly increased in CD4 T cells in both ICU and non-ICU patients as compared to healthy individuals $(P<0.001)$ (Figure $1 \mathrm{~b})$, suggesting a recent exposure to cytokines or growth factors $^{24}$. Of note, several signaling pathways such as pNF-kb, pCREB, pERK1/2, pS6 and p38 were increased but no significant difference was observed between ICU and non-ICU patients $(P>0.05)$ (Figure 1b).

186

\section{Cytokine signatures in ICU versus non-ICU patients}



severity ${ }^{11,12}$. We determined whether a cytokine signature could help identifying at the time of hospital admission patients with severe COVID-19 requiring ICU admission. We therefore assessed the serum levels of a large panel $(n=49)$ of mediators including cytokines, soluble cytokine receptors, chemokines and growth factors in blood samples collected at the time of admission in 43 ICU patients and 55 non-ICU patients enrolled in the 'discovery' cohort (LUH1). The serum concentration of these 49 markers of inflammation were compared to the levels measured in 450 sera collected from healthy individuals that were used as normal reference values. Serum levels of a large panel of cytokines, chemokines and growth factors were markedly increased in ICU and non-ICU patients compared to those of healthy individuals $(P<0.05)$ (Figure 2). However, serum levels of CCL4, CCL11, nerve growth factor- $\beta$ (NGF$\beta$ ), epidermal growth factor (EGF), fibroblast growth factor-2 (FGF-2) and placental growth factor-1 (PIGF-1) were significantly decreased in both ICU and non-ICU patients compared to healthy individuals $(P<0.05$ to $P<0.001)$ (Figure 2$)$. Of note, serum levels of IL-1RA, IL-1 $\beta$, IL-6, IL-10, IL-15, CCL2, CCL4, CXCL9, CXCL10, CXCL13, HGF, LIF and VEGF-A were significantly increased in ICU versus non-ICU patients $(P<0.001)$ (Figure 2).

To better define the serum factor signatures potentially differentiating ICU from nonICU individuals, the levels of the 49 serum factors were compared between groups using Kruskal-Wallis test corrected for multiple comparison. For each candidate marker, the optimal cutpoint to distinguish between ICU and non-ICU patients was determined using the cutpt command of Stata, applying the Liu method to maximize the product of the sensitivity and specificity. Based on the cutpoints, the candidate markers were dichotomized into lower and higher or equal to the cutpoint and the area under the receiver-operating curve (AUC) (Figure 3), the sensitivity, specificity, positive and negative predictive values and the likelihood ratio

212 (Table 1) were computed. This analysis identified a panel of 13 serum factors (IL-10, CCL2, 213 CCL4, CXCL13, IL-1RA, IL-6, IL-15, VEGF-A, CXCL9, CXCL10, IL-1 $\beta$, LIF and HGF) differently distributed between ICU and non-ICU patients (Supplemental Figure 3). Based on these analyses, HGF and CXCL13 showed the best sensitivity (88.6\% for both HGF and CXCL13) and specificity (81.5\% for HGF and 79.6\% for CXCL13) to discriminate between ICU and non-ICU patients (Table 1 and Figure 3). More importantly, the positive predictive values (PPV) were $79.6 \%$ for HGF and $78 \%$ for CXCL-13 and the negative predictive values (NPV) were $98.9 \%$ for HGF and $89.6 \%$ for CXCL13.

We then performed a blinded evaluation of the serum levels of the 49 cytokines in samples collected from patients enrolled in two independent 'validation' COVID-19 cohorts 
of the FCS ( $n=62$ patients) and of the LUH- 2 cohort ( $n=47$ patients). The LHU- 2 cohort was enrolled based on the same criteria of the LUH-1 cohort. Demographic and clinical data of the FCS 'validation' cohort are summarized in Supplemental Table 4. Admission to the ICU for the FCS followed the recommendations of the guidelines of the French Haute Autorite de Santé. We then applied the cut-points values for the 13 serum factors (IL-10, CCL2, CCL4, CXCL13, IL-1RA, IL-6, IL-15, VEGF-A, CXCL9, LIF, IL-1 $\beta$, CXCL10 and HGF) defined in the 'discovery' cohort. Following unblinding of the FCS, increased levels of HGF and CXCL13 predicted ICU admission in $27(87.0 \%)$ of 31 patients and non-ICU admission in 29 (93.5\%) of 31 patients. Following unblinding of the LUH-2 cohort, ICU admission was predicted in 34 (94.4\%) of 36 patients and internal medicine ward admission in 10 (90.9\%) of 11 patients. ROC and AUC analyses confirmed the hierarchy amongst the 13 selected cytokines in discriminating between ICU and non-ICU patients in the FCS and LUH-2 validation cohorts (Supplemental Figure 4 and Table 2).

Thus, HGF and CXCL13 were the best predictors of COVID-19 severity and ICU admission. Interestingly, the combination of HGF and CXCL13 further improved their discriminative power for ICU admission in the 'discovery' and 'validation' cohorts (Table 3). The performance of the combination of the two cytokines was as follows: sensitivity $=79.1 \%$, specificity $=94.5 \%, \mathrm{PPV}=91.9 \%, \mathrm{PNV}=85.2 \%$ in the 'discovery' cohort, sensitivity = $81.8 \%$, specificity $=94.4 \%, \mathrm{PPV}=81.8 \%, \mathrm{PNV}=94.4 \%$ in the LUH-2 'validation' cohort, and sensitivity $=87.1 \%$, specificity $=93.5 \%, \mathrm{PPV}=93.1 \%, \mathrm{PNV}=87.9 \%$ in the France COVID-19 Study 'validation' cohort (Table 3).

We next assessed the potential of the 13 serum factors (IL-10, CCL2, CCL4, CXCL13, IL-1RA, IL-6, IL-15, VEGF-A, CXCL9, LIF, IL-1 $\beta$, CXCL10 and HGF) and their relative cutpoint values to predict 30-day mortality among the COVID-19 patients enrolled in the combined LUH-1, LUH-2 and FCS cohorts. Among the initial 207 patients, vital status at 30 days was available for 197 and 186 had data allowing for survival analysis. The associations between categories of markers and vital status were assessed by chi-square; survival analysis was performed via a multilevel survival model using a Weibull distribution and results were expressed as multivariable-adjusted hazards ratio (HR) with a 95\% confident interval (CI). Overall, 18 patients died, 17 of whom had high levels of the combination of HGF and CXCL13 $(\mathrm{P}=0.006)$; survival analysis showed that patients with the combination of HGF and CXCL13 had a 8.80-fold higher likelihood of dying ( $\mathrm{P}=0.054)$ (Table 4).

\section{Discussion}


The hallmark of severe COVID-19 is an acute respiratory distress syndrome (ARDS) with respiratory failure requiring mechanical ventilation in $10 \%$ to $24 \%$ of hospitalized patients. A large number of studies have drawn attention to systemic immune activation involving both the innate and adaptive arms of the host immune system ${ }^{11,12,20,25,26}$. The relevance in COVID-19 of the massive release of a large number of soluble mediators including cytokines, cytokine receptors, growth factors and chemokines has been thoroughly discussed in a recent 'Opinion' article ${ }^{27}$. The article has highlighted that the pathophysiology of the COVID-19 cannot be explained solely on the basis of the increase in a few inflammatory cytokines such as IL-6 and TNF. It remains unclear to what extent the increase of circulating mediators drives the pathogenesis of severe COVID-19.

A large number of studies have been carried out to better understand the pathophysiology of COVID-19 and identify predictive markers of disease severity in the early symptomatic phase of infection ${ }^{11,12,20,25,26}$. Consistent with these studies ${ }^{11,12,25,26}$, we observed that several cellular markers of activation and differentiation of blood T, B, monocyte and DC cell populations were abnormal in SARS-CoV2 infected patients compared to healthy individuals. However, none of these cellular markers can discriminate between severe and moderate COVID-19. Of note, we have also shown in SARS-CoV2 patients an increase of Th1 and Th1/Th17 CD4 $\mathrm{T}$ cell lineages and a decrease in Th2 cells supporting the inflammatory profile of the $\mathrm{T}$ cell response associated with COVID-19. Furthermore, the increase in signaling pathways such as pNF- $\mathrm{kb}, \mathrm{pCREB}, \mathrm{pERK} 1 / 2, \mathrm{pS} 6$ and p38 is consistent with the cytokinemediated activation of the different pro-inflammatory CD4 T cell lineages.

Consistent with previous studies ${ }^{11,12,26}$, we confirmed the increase in a large number of soluble mediators in patients with COVID-19. However, the results of the present study provide a substantial advance in the understanding of the pathophysiology of COVID-19 and in the identification of predictive markers of the severity of the SARS-CoV2 infection. Two recent studies attempted to identify markers of disease severity. In one study ${ }^{11}$, only a small number $(n=4)$ of cytokines were measured and IL-6, TNF and IL-8 were identified as markers of severity of COVID-19 as measured by mortality. The study was conducted in a large number of patients but was unable to predict the severity of the disease at the time of hospital admission. In two studies, conducted on a small number $\left(n=49^{12}\right.$ and $\left.n=40^{13}\right)$ of patients, HGF in addition to other markers was proposed to serve as a marker of severity of COVID-19.

It is important to underscore that in our study the serum samples were collected at the time of hospital admission in the 'discovery' and in the two 'validation' cohorts. The timing of 
sampling is critical because serum cytokine levels can change substantially as the infection progresses. We have shown that, among the 49 soluble mediators measured, two cytokines, HGF and CXCL13, are the best predictors of the need for ICU hospitalization for COVID-19 patients.

HGF is a pleiotropic cytokine produced by mesenchymal cells and macrophages. It is required for normal embryogenesis and development ${ }^{28,29}$ of several organs including the lung ${ }^{30}$. In adults, HGF is produced following injury of the lung tissue and promotes tissue repair ${ }^{31-34}$. HGF promotes lung tissue repair through the inhibition of apoptosis of lung epithelial and endothelial cells, and by counteracting a number of pro-apoptotic and pulmonary fibrosis factors such as TGF- $\beta$, IL-1 $\beta$, IL-8, TNF- $\alpha$, the basic fibroblastic factor, the insulin-like growth factor and the platelet-derived growth factor ${ }^{35-44}$. It has been proposed that the anti-apoptotic activity of $\mathrm{HGF}$ is due in particular to the activation of three signaling pathways, i.e. ERK/MAPK, PI3K/Akt, and STAT3 ${ }^{45-47}$.

HGF may play also a central role in the regulation of inflammation. A number of proinflammatory cytokines such as IFN- $\gamma, \mathrm{IL}-1 \alpha / \beta$ and TNF- $\alpha$ induce HGF expression as well as activated $\mathrm{T}$ cells ${ }^{48,49}$ while glucocorticoids and TGF- $\beta$ inhibit HGF production ${ }^{50}$. HGF may induce monocyte-macrophage activation ${ }^{51}$, B cell homing ${ }^{52}$, and modulation of DC functions ${ }^{53}$. HGF exerts predominantly an anti-inflammatory role through the decrease production of IL-6 and increase production of IL-10 $0^{54,55}$, by preventing the differentiation of inflammatory $\mathrm{T}$ cell lineages through the suppression of DC-mediated IL-12p70 production ${ }^{55,56}$, and by favoring

310 Tregs maturation ${ }^{55,57}$. Finally, HGF produced by follicular DC is a positive regulator of growth and survival of B cells and plasma cells ${ }^{49,58}$.

CXCL13 plays a central physiological role in the organization of secondary lymphoid tissue structure of primary and secondary follicles and thus of B cell maturation ${ }^{59}$. CXCL13 is a pro-inflammatory cytokine involved in several pathological conditions and the finding of increased levels in tissue and/or in serum corresponds to varying degrees of inflammation. CXCL13 serum levels have been found increased in several uncontrolled infectious disease such as in viremic HIV infection, in a variety of autoimmune diseases, and in both hematological and solid tumors (reviewed $\mathrm{in}^{59}$ ). Interestingly, increased serum levels and tissue expression of CXCL13 have been initially found to be associated with idiopathic pulmonary fibrosis $^{60,61}$ and recently in several interstitial lung diseases including idiopathic interstitial pneumonia and interstitial pneumonia with autoimmune features ${ }^{62}$. The increased levels of 

some of the signaling pathways such as ERK/MAPK and PI3/AKT (reviewed in ${ }^{59}$ ).

Based on the biology of HGF, our observation of increased serum levels early in symptomatic infection and its association with ICU hospitalization is likely an indicator of an ongoing severe respiratory syndrome associated with interstitial pneumonia. Up-regulation of HGF is the host's physiological counter-regulatory immune response to reduce inflammation, to limit lung tissue injury and to promote tissue repair. Consistent with this view, over $90 \%$ of non-ICU patients with a moderate respiratory syndrome had low levels of HGF. Of note, HGF may exert its anti-inflammatory property through IL-10. Interestingly, IL-10 was one of the thirteen cytokines found to discriminate ICU from non-ICU patients.

With regard to CXCL13, the early increase in the symptomatic severe COVID-19 may also reflect the potent host immune response to promote maturation of B cell and antibody response in order to achieve rapid control of the virus replication and virus clearance. However, the persistence of elevated levels of CXCL13 in the lung tissue and serum may be detrimental and responsible for fueling the inflammation and promoting lung fibrosis.

Of note, we have demonstrated that the combined use of HGF and CXCL13 provides a powerful immune signature discriminating between ICU and non-ICU patients at hospital admission with positive and negative predictive values ranging from $81.8 \%$ to $93.1 \%$ and $85.2 \%$ to $94.4 \%$ in the 3 cohorts, and predicting the occurrence of death during patient followup. Therefore, the combined assessment of the two cytokines is a valuable tool in the clinical management of patients with acute SARS-CoV-2 infection.

In conclusion, the present study provides novel insights in the early pathophysiological events associated with severe COVID-19 and identified HGF and CXCL13 as critical pathogenic biomarkers of disease severity and best predictors of ICU admission and death.

\section{Methods}

349 Study group, ethics statement. Eighty-eight ICU and one hundred twenty five non-ICU hospitalized PCR-confirmed SARS-CoV2 infected individuals were enrolled in the present study. No statistical method was used to predetermine sample size. The sample size was estimated based on a previously published study ${ }^{25}$. The present study was approved by the ethical commission (CER-VD) and all subjects provided a written informed consent. As inclusion criteria, only patients with a positive SARS-CoV2 PCR were enrolled. As exclusion criteria, pregnant women were not enrolled. Serum and blood samples were also collected from 450 healthy individuals during the pre-pandemic period. The exclusion criteria were sign of 
acute or chronic viral hepatitis (HAV, HBV, HCV and HEV), prior diagnosis of autoimmune disease (e.g. rheumatoid arthritis, psoriasis, SLE), prior diagnosis of primary or secondary immunodeficiency (e.g. HIV infection) and current or past (last 4 weeks) use of medications that are known modify the immune response.

Assessment of serum immune signatures. Serum concentration of cytokines and soluble cytokine receptors i.e. IL-1 $\alpha$, IL-1RA, IL-1 $\beta$, IL-2 , IL-4, IL-5, IL-6, IL-7, IL-9, IL-10, IL12p70, IL-13, IL-15, IL-17A, IL-18, IL-21, IL -22, IL-23, IL-27, IL-31, IFN- $\alpha$, IFN- $\gamma$ and TNF, chemokines i.e. CCL2, CCL3, CCL4, CCL5, CCL11, CXCL1, CXCL8, CXCL9, CXCL10, CXCL12, CXCL13 and TNF- $\beta$ and growth factors i.e. NGF-beta, BDNF, EGF, FGF-2, HGF, LIF, PDGF-BB, PIGF-1, SCF, VEGF-A, VEGF-D, BAFF, GM-CSF and G-CSF were determined by multiplex bead assay as previously described ${ }^{63}$. The upper normal values for each marker were defined based on the results obtained in the 450 sera collected from healthy individuals (mean +2 standard deviations).

Immune profiling of circulating cell populations by mass cytometry. Blood samples (200 $\mu 1$ ) were first incubated (30 min; RT) with metal-conjugated antibodies directed against CD3, CD7, CD45, CCR7, CXCR3, CXCR5, and $\gamma \delta$ TCR (c.f. antibodies section; Panel 1). Cells were then fixed (5 min; RT) with PBS 2.4\% PFA and lysed (15 minutes, RT) using Bulklysis solution (Cytognos) and washed (PBS, 0.5\% BSA, Sodium azide 0.02\%). Cells were then incubated (30 min; RT) with the remaining metal-conjugated monoclonal antibodies (c.f. antibodies section). Cells were then washed (PBS, $0.5 \%$ BSA, Sodium azide $0.02 \%$ ) and fixed ( 5 min; RT) with PBS $2.4 \%$ PFA. Cells were stained (1 hour; RT) with DNA intercalator $(1 \mu \mathrm{M}$ Cell-ID Intercalator, Fluidigm/DVS Science) in PBS, 0.5\% BSA, sodium azide 0.02\%, 0.3\% saponin, $1.6 \%$ PFA.

Evaluation of CD4 T cell lineage distribution by mass cytometry. Blood samples $(100 \mu \mathrm{l})$ were first incubated (30 min; RT) with metal-conjugated antibodies directed against CD8, CD4, CCR4, CD127, CCR6, CXCR3, CCR9, CCR7, CXCR5, CCR5 and CD45 (c.f. antibodies section). Cells were then fixed (5 min; RT) with PBS 2\% PFA and lysed (15 minutes, RT) using Bulklysis solution (Cytognos) and washed (PBS, 0.5\% BSA, Sodium azide 0.02\%). Cells were then incubated (30 min; RT) with the metal-conjugated monoclonal antibodies directed against CD3, CD44, CD25, CCR6, CXCR5, CD38, TIGIT, 2B4, PD1, CD27, CD69, CD45RO, CD127, CD16, CD31, CD95, CD57, NKG2D, CD45RA, HLADR, PDL1, CD151, CD40L, ICOS, LAG3, OX40 (c.f. antibodies section; Panel 2). Cells were then washed (PBS, 0.5\% BSA, Sodium azide 0.02\%) and fixed (5 min; RT) with PBS 2.4\% PFA. Cells were then 
permeabilized (30 min; $4^{\circ} \mathrm{C}$ ) (Foxp3 Fixation/Permeabilization Kit; eBioscience) then washed and stained $\left(30 \mathrm{~min} ; 4^{\circ} \mathrm{C}\right)$ with the metal-conjugated monoclonal antibodies directed against Tbet, Ki67, Bcl2, RoryT, Gata3, FoxP3 (c.f. antibodies section; Panel 2). Cells were then washed (PBS, 0.5\% BSA, 0.3\% saponin, Sodium azide 0.02\%). Cells were stained (1 hour; RT) with DNA intercalator ( $1 \mu \mathrm{M}$ Cell-ID Intercalator, Fluidigm/DVS Science) in PBS, $0.5 \%$ BSA, sodium azide $0.02 \%, 0.3 \%$ saponin, $1.6 \%$ PFA.

Assessment of the CD4 T cell phospho-protein signaling profile by mass cytometry. Blood samples (200 $\mu \mathrm{l}$ ) were first fixed (5 min; RT) with PBS 2.4\% PFA and lysed (15 minutes, RT) using Bulklysis solution (Cytognos) and washed (PBS, 0.5\% BSA, Sodium azide 0.02\%). Cells were then incubated (30 min; RT) with the metal-conjugated monoclonal antibodies directed against CD3, CD45, CD8, CD4, CD19, CD1c, CD69, CD31, CD86, CD7, CD39, CD56, CD123, CD21, CD27, CD14, CD11c, CD62L, CD161, CD20, CD38, CD45RA, CD15, CD141, HLA-DR, CD57 and CD16 (c.f. antibodies section; Panel 3). Cells were then washed (PBS, 0.5\% BSA, Sodium azide 0.02\%) and fixed (5 min; RT) with PBS 2.4\% PFA. Cells were then permeabilized $\left(30 \mathrm{~min} ; 4^{\circ} \mathrm{C}\right.$ ) (Foxp3 Fixation/Permeabilization Kit; eBioscience) then washed and stained $\left(30 \mathrm{~min} ; 4^{\circ} \mathrm{C}\right)$ with the metal-conjugated monoclonal antibodies directed against pSTAT1, pSTAT3, pSTAT5, p38, pMAPKAPK2, pNFkb, Ki67, pERK1/2, pS6, pCREB, (c.f. antibodies section; Panel 3). Cells were then washed (PBS, 0.5\% BSA, 0.3\% saponin, Sodium azide $0.02 \%)$. Cells were stained (1 hour; RT) with DNA intercalator $(1 \mu \mathrm{M}$ Cell-ID Intercalator, Fluidigm/DVS Science) in PBS, $0.5 \%$ BSA, sodium azide $0.02 \%, 0.3 \%$ saponin, $1.6 \%$ PFA. Labeled samples were acquired on a Helios instrument using a flow rate of $0.030 \mathrm{ml} / \mathrm{min}$. Data were analysed using FlowJo software (v10.2). At least 500,000 events were acquired for each sample.

413 Antibodies. The following antibodies were used for mass cytometry experiments. Panel 1: 111Cd-conjugated anti-CD141 (1A4), 113In-conjugated anti-CD8 (RPA-T8), 115Inconjugated anti-CD4 (RPA-T4), 116Cd-conjugated anti-IgA2 (A9604D2), 141Pr-conjugated anti-CD45 (HI30), 142Nd-conjugated anti-CD19 (HIB19), 143Nd-conjugated anti-ICOS (C398.4A), 144Nd-conjugated anti-IgG3 (HP6047), 145Nd-conjugated anti-CD31/PECAM-1 (WM59), 146Nd-conjugated anti-IgD (IA6-2), 147Sm-conjugated anti-CD7 (CD7-6B7), 148Nd-conjugated anti-IgA1 (B3506B4), 149Sm-conjugated anti-CD127 (A019D5), 150Ndconjugated anti-IgG1 (G17-1), 151Eu-conjugated anti-CD123 (6H6), 152Sm-conjugated antiCD21 (BL13), 153Eu-conjugated anti-CD62L (DREG-56), 154Sm-conjugated anti-CD3 (UCHT1), 155Gd-conjugated anti-CD27 (L128), 156Gd-conjugated anti-TCR $\gamma$ (gamma)/ $\delta$ (delta) (B1), 158Gd-conjugated anti-CD10 (HI10a), 159Tb-conjugated anti-CD197/CCR7 
(G043H7), 160Gd-conjugated anti-CD14 (M5E2), 161Dy-conjugated anti-CD1c (L161), 162Dy-conjugated anti-CD11c (Bu15), 163Dy-conjugated anti-CD183/CXCR3 (G025H7), 164Dy-conjugated anti-CD185/CXCR5 (51505), 165Ho-conjugated anti-CD45RO (UCHL1), 166Er-conjugated anti-CD24 (ML5), 167Er -conjugated anti-CD38 (HIT2), 168Er-conjugated anti-CD66b (G10F5), 169Tm-conjugated anti-CD25 (2A3), 170Er-conjugated anti-CD45RA (HI100), 171 Yb-conjugated anti-CD20 (2H7), 172Yb-conjugated anti-IgM (MHM-88), 173Yb-conjugated anti-TCR $\alpha$ (alpha)/ $\beta$ (beta) (T1089.A-31), 174Yb-conjugated anti-HLADR (L243), 175Lu-conjugated anti-CD279/PD-1 (EH12.2H7), 176Yb-conjugated anti-CD56 (HCD56), 198Pt-conjugated anti-IgG2 (HP6002), 209Bi-conjugated anti-CD16 (3G8), 112Cdconjugated anti-CD69 (FN50), 106Cd-conjugated anti-CCR6 (11A9), 194Pt-conjugated antiCCR4 (L291H4) and 191Ir was used to label DNA. Antibodies against TCR $\alpha$ (alpha)/ $\beta$ (beta), IgG1, CD66b, CCR6 and CD141 were purchased from BD. Antibodies against TCR $\gamma$

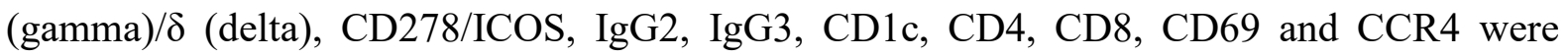
purchased from Biolegend. Antibodies against $\operatorname{IgA} 1$ and $\operatorname{IgA} 2$ were purchased from SouthernBiotech. All were conjugated with Maxpar® X8 Antibody Labeling Kit except IgA2 and CD141 who were labelled using Maxpar MCP9 Antibody Labeling Kit. All other antibodies were purchased from Fluidigm/DVS. Panel 2: 113In-conjugated anti-CD8 (RPAT8), 115In-conjugated anti-CD4 (RPA-T4), 149Sm-conjugated anti-CCR4 (L291H4), 176Ybconjugated anti-CD127 (A019D5), 141Pr-conjugated anti-CCR6 (G034E3), 154Smconjugated anti-CXCR3 (G025H7), 168Er-conjugated anti-CCR9 (L053E8), 159Tbconjugated anti-CCR7 (G043H7), 167Er-conjugated anti-CXCR5 (J252D4), 144Ndconjugated anti-CCR5 (NP-6G4), 106Cd-conjugated anti-CD45 (HI30), 111Cd-conjugated anti-CD3 (UCHT1), 142Nd-conjugated anti-CD44 (IM7), 158Gd-conjugated anti-CD25 (MA251), 141Pr-conjugated anti-CCR6 (G034E3), 163Dy-conjugated anti-CD38 (HIT2), 153Euconjugated anti-TIGIT (MBSA43), 147Sm-conjugated anti-2B4 (C1.7), 151Eu-conjugated anti-PD1 (EH12.2H7), 155Gd-conjugated anti-CD27 (L128), 162Dy-conjugated anti-CD69 (FN50), 164Dy-conjugated anti-CD45RO (UCHL1), 209Bi-conjugated anti-CD16 (3G8), 145Nd-conjugated anti-CD31 (WM59), 161Dy-conjugated anti-CD95 (DX2), 194Ptconjugated anti-CD57 (NK-1), 166Er-conjugated anti-NKG2D (ON72), 170Er-conjugated anti-CD45RA (HI100), 174Yb-conjugated anti-HLADR (L243), 148Nd-conjugated antiPDL1 (29E.2A3), 171Yb-conjugated anti-CD151 (50/6), 152Sm-conjugated anti-CD40L (TRAP1), 143Nd-conjugated anti-ICOS (C398.4A), 172Yb-conjugated anti-LAG3 (874501), 150Nd-conjugated anti-OX40 (ACT35), 160Gd-conjugated anti-Tbet (4B10), 165Hoconjugated anti-Ki67 (Ki67), 169Tm-conjugated anti-Bcl2 (100), 175Lu-conjugated anti- 
RoryT (AFKJS-9), 146Nd-conjugated anti-Gata3 (TWAJ), 156Gd-conjugated anti-FoxP3 (PCH101) and 191Ir was used to label DNA. Antibodies against CD45, CD8, CD4, CD44, ICOS, 2B4, PD-1, CXCR3, CD25, CCR7, CD38, Ki67, CXCR5, Bcl2 were purchased from Biolegend. Antibodies against CD3, CD40L (CD154), CD57 and CD151 were purchased from BD. Antibodies against Gata3, FoxP3, CD95(FAS) and RoryT were purchased from eBiosciences. Antibody against LAG3 was purchased from R\&D Systems. All were conjugated with Maxpar® X8 Antibody Labeling Kit except CD45 and CD3 who were labelled using Maxpar MCP9 Antibody Labeling Kit. All other antibodies were purchased from Fluidigm/DVS. Panel 3: 154Sm -conjugated anti-CD3 (UCHT1), 106Cd -conjugated antiCD45 (HI30), 113In -conjugated anti-CD8 (RPA-T8), 115In -conjugated anti-CD4 (RPA-T4), 142Nd -conjugated anti-CD19 (HIB19), 143Nd -conjugated anti-CD1c (L161) , 144Nd conjugated anti-CD69 (FN50), 145Nd -conjugated anti-CD31 (WM59), 146Nd -conjugated anti-CD86 (GL-1) , 147Sm -conjugated anti-CD7 (CD7-6B7), 148Nd -conjugated anti-CD39 (A1) , 149Sm -conjugated anti-CD56 (HCD56), 150Nd -conjugated anti-pSTAT5 (47), 151Euconjugated anti-CD123 (6H6), 152Sm -conjugated anti-CD21 (BL13), 153Eu -conjugated antipSTAT1 [Y701] (58D6), 155Gd -conjugated anti-CD27 (L128), 156Gd -conjugated anti-p38 [T180/Y182] (D3F9), 158Gd -conjugated anti-pSTAT3 (4/P-Stat3), 159Tb -conjugated antipMAPKAPK2 (27B7), 160Gd -conjugated anti-CD14 (M5E2), 162Dy -conjugated antiCD11c (Bu15), 163Dy -conjugated anti-CD62L (DREG-56), 164Dy -conjugated anti-CD161 (HP3G10), 165Ho -conjugated anti-pNFkb (K10895.12.50), 166Er -conjugated anti-CD20 (2H7), 167Er -conjugated anti-CD38 (HIT2), 168Er -conjugated anti-Ki67 (Ki67) , 169Tm conjugated anti-CD45RA (HI100) , 171Yb -conjugated anti-pERK1/2 [T202/Y204] (D13.14.4E), 172 Yb -conjugated anti-CD15 [SSEA-1] (W6D3), 173Yb -conjugated antiCD141 (1A4), 174Yb -conjugated anti-HLA-DR (L243), 175Lu -conjugated anti-pS6 (N7548), 176Yb -conjugated anti-pCREB (87G3), 194Pt -conjugated anti-CD57 (NK-1), 209Bi -conjugated anti-CD16 (3G8) and 191Ir was used to label DNA. Antibodies against Ki67, CD45, CD8a, CD4, CD1c, CD69, CD86, CD39, CD56, CD62L and CD45RA were purchased from Biolegend. Antibodies against NF-kB p65, CD20, CD141 and CD57 were purchased from BD. All were conjugated with Maxpar® X8 Antibody Labeling Kit except CD45 which was labelled using Maxpar MCP9 Antibody Labeling Kit. All other antibodies were purchased from Fluidigm/DVS.

489 Statistical analyses. Statistical analyses were conducted using R version (v.3.6.3) (The R 490 Foundation for Statistical Computing) and Stata version 16.1 (Stata Corp, College Station, TX, 491 USA). Inter-group clinical data comparisons were performed using chi-square or Fisher's exact 
492 test for categorical variables and Kruskal-Wallis test for continuous variables. Descriptive 493 values were presented as violin plots for continuous variables. Serum marker levels and mass 494 cytometry cell population values were $\log 10$ transformed for statistical analysis. Statistical 495 analyses were conducted using $\mathrm{R}$ version (v.3.6.3) (The R Foundation for Statistical 496 Computing). Statistical significance ( $P$ values) was obtained using Kruskal-Wallis test. 497 Bonferroni's correction was applied for multiple comparison. Serum markers whose p-value 498 was below the threshold were then considered as candidates for diagnosis of severe (ICU) 499 cases. For each candidate marker, the optimal cutpoint to distinguish between ICU and non500 ICU patients was computed using the cutpt command of Stata version 16.1 (Stata Corp, College 501 Station, TX, USA) and default settings (i.e. maximization of the sensitivity $\mathrm{x}$ specificity 502 product). Based on the results, the candidate markers were dichotomized into lower than and 503 higher or equal to the cutpoint, and the area under the receiver-operating curve (AUC), the 504 sensitivity, specificity, positive and negative predictive values and the positive likelihood ratio 505 were computed using the roccomp and the diagt commands of Stata. The two markers 506 displaying the best AUCs were then combined into a binary variable (both values high and 507 other) and considered for classification of the patients. The clinical relevance of the two 508 markers was checked by multivariable analysis using stepwise forward logistic regression 509 using a p-value for entry=0.05 and a p-value for removal=0.10. Of the initial 207 patients, 197 510 had vital data at follow-up and 186 had data allowing for survival analysis. Bivariate analysis 511 of the associations between categories of markers and vital status (death/alive) were assessed 512 using chi-square. Survival analysis was conducted using Cox proportional hazards regression, 513 unadjusted or adjusted for age. A second survival analysis was conducted using a multilevel 514 survival model using a Weibull distribution, where patients were nested within each cohort, 515 and adjusting for age (continuous) and ICU stay (yes/no). For the survival analysis, results 516 were expressed as multivariable-adjusted hazards ratio (HR) and 95\% CI. 


\section{References}

5181 Azkur, A. K. et al. Immune response to SARS-CoV-2 and mechanisms of immunopathological changes

in COVID-19. Allergy 75, 1564-1581, doi:10.1111/all.14364 (2020).

2 Fajgenbaum, D. C. \& June, C. H. Cytokine Storm. N Engl J Med 383, 2255-2273, doi:10.1056/NEJMra2026131 (2020).

3 Sinha, P. et al. Prevalence of phenotypes of acute respiratory distress syndrome in critically ill patients with COVID-19: a prospective observational study. The Lancet. Respiratory medicine 8, 1209-1218, doi:10.1016/s2213-2600(20)30366-0 (2020).

4 Mehta, P. et al. COVID-19: consider cytokine storm syndromes and immunosuppression. Lancet (London, England) 395, 1033 (2020).

5 Catanzaro, M. et al. Immune response in COVID-19: addressing a pharmacological challenge by targeting pathways triggered by SARS-CoV-2. Signal Transduction and Targeted Therapy 5, 84, doi:10.1038/s41392-020-0191-1 (2020).

6 Leisman, D. E. et al. Cytokine elevation in severe and critical COVID-19: a rapid systematic review, meta-analysis, and comparison with other inflammatory syndromes. The Lancet. Respiratory medicine 8, 1233-1244, doi:10.1016/s2213-2600(20)30404-5 (2020).

$7 \quad$ Wong, C. et al. Plasma inflammatory cytokines and chemokines in severe acute respiratory syndrome. 136, 95-103 (2004).

8 Zhang, Y. et al. Analysis of serum cytokines in patients with severe acute respiratory syndrome. $\mathbf{7 2}$, 4410-4415 (2004).

9 Hue, S. et al. Uncontrolled Innate and Impaired Adaptive Immune Responses in Patients with COVID19 Acute Respiratory Distress Syndrome. Am J Respir Crit Care Med 202, 1509-1519, doi:10.1164/rccm.202005-1885OC (2020).

10 Huang, C. et al. Clinical features of patients infected with 2019 novel coronavirus in Wuhan, China. 395, 497-506 (2020).

11 Del Valle, D. M. et al. An inflammatory cytokine signature predicts COVID-19 severity and survival. Nature Medicine 26, 1636-1643, doi:10.1038/s41591-020-1051-9 (2020).

12 Meizlish, M. L. et al. A neutrophil activation signature predicts critical illness and mortality in COVID19. medRxiv, 2020.2009.2001.20183897, doi:10.1101/2020.09.01.20183897 (2020).

13 Haljasmägi, L. et al. Longitudinal proteomic profiling reveals increased early inflammation and sustained apoptosis proteins in severe COVID-19. Scientific reports 10, 20533, doi:10.1038/s41598-02077525-w (2020).

14 Herold, S. et al. Lung epithelial apoptosis in influenza virus pneumonia: the role of macrophageexpressed TNF-related apoptosis-inducing ligand. $J$ Exp Med 205, 3065-3077, doi:10.1084/jem.20080201 (2008).

15 Palm, N. W. \& Medzhitov, R. J. N. m. Not so fast: adaptive suppression of innate immunity. 13, 11421144 (2007).

16 Jiang, Y. et al. Characterization of cytokine/chemokine profiles of severe acute respiratory syndrome. 171, 850-857 (2005).

17 Hu, B., Guo, H., Zhou, P. \& Shi, Z.-L. Characteristics of SARS-CoV-2 and COVID-19. Nature Reviews Microbiology, doi:10.1038/s41579-020-00459-7 (2020).

18 Mathew, D. et al. Deep immune profiling of COVID-19 patients reveals distinct immunotypes with therapeutic implications. Science 369, eabc8511, doi:10.1126/science.abc8511 (2020).

19 Thevarajan, I. et al. Breadth of concomitant immune responses prior to patient recovery: a case report of non-severe COVID-19. Nature Medicine, doi:10.1038/s41591-020-0819-2 (2020).

20 Mathew, D. et al. Deep immune profiling of COVID-19 patients reveals distinct immunotypes with therapeutic implications. Science, eabc8511, doi:10.1126/science.abc8511 (2020).

21 Grifoni, A. et al. Targets of T cell responses to SARS-CoV-2 coronavirus in humans with COVID-19 disease and unexposed individuals. Cell (2020).

22 De Biasi, S. et al. Marked T cell activation, senescence, exhaustion and skewing towards TH17 in patients with COVID-19 pneumonia. Nature communications 11, 1-17 (2020).

23 Weatherhead, J. E., Clark, E. H., Vogel, T. P., Atmar, R. L. \& Kulkarni, P. A. Inflammatory syndromes associated with SARS-CoV-2 infection: dysregulation of the immune response across the age spectrum. The Journal of Clinical Investigation, doi:10.1172/JCI145301 (2020).

24 Seif, F. et al. The role of JAK-STAT signaling pathway and its regulators in the fate of T helper cells. Cell communication and signaling : CCS 15, 23, doi:10.1186/s12964-017-0177-y (2017).

25 Laing, A. G. et al. A dynamic COVID-19 immune signature includes associations with poor prognosis. Nat Med 26, 1623-1635, doi:10.1038/s41591-020-1038-6 (2020). 
Kermali, M., Khalsa, R. K., Pillai, K., Ismail, Z. \& Harky, A. The role of biomarkers in diagnosis of

27 Sinha, P., Matthay, M. A. \& Calfee, C. S. Is a "Cytokine Storm" Relevant to COVID-19? JAMA Internal (2020) Medicine 180, 1152-1154, doi:10.1001/jamainternmed.2020.3313 (2020).

28 Sonnenberg, E., Weidner, K. M. \& Birchmeier, C. Expression of the met-receptor and its ligand, HGFSF during mouse embryogenesis. Exs 65, 381-394 (1993).

Birchmeier, C. \& Gherardi, E. Developmental roles of HGF/SF and its receptor, the c-Met tyrosine kinase. Trends Cell Biol 8, 404-410, doi:10.1016/s0962-8924(98)01359-2 (1998).

Yamamoto, H. et al. Epithelial-vascular cross talk mediated by VEGF-A and HGF signaling directs primary septae formation during distal lung morphogenesis. Dev Biol 308, 44-53, doi:10.1016/j.ydbio.2007.04.042 (2007).

Yanagita, K. et al. Hepatocyte growth factor may act as a pulmotrophic factor on lung regeneration after acute lung injury. J Biol Chem 268, 21212-21217 (1993).

Adamson, I. Y. \& Bakowska, J. Relationship of keratinocyte growth factor and hepatocyte growth factor levels in rat lung lavage fluid to epithelial cell regeneration after bleomycin. The American journal of pathology 155, 949-954, doi:10.1016/S0002-9440(10)65194-2 (1999).

Liu, X. L., Sato, S., Dai, W. \& Yamanaka, N. The protective effect of hepatocyte growth-promoting factor (pHGF) against hydrogen peroxide-induced acute lung injury in rats. Medical electron microscopy : official journal of the Clinical Electron Microscopy Society of Japan 34, 92-102, doi:10.1007/s007950170003 (2001).

Matsumoto, K. \& Nakamura, T. Roles of HGF as a pleiotropic factor in organ regeneration. Exs 65, 225249 (1993).

Border, W. A. \& Noble, N. A. Transforming growth factor beta in tissue fibrosis. N Engl J Med 331, 1286-1292, doi:10.1056/nejm199411103311907 (1994).

Broekelmann, T. J., Limper, A. H., Colby, T. V. \& McDonald, J. A. Transforming growth factor beta 1 is present at sites of extracellular matrix gene expression in human pulmonary fibrosis. Proc Natl Acad Sci U S A 88, 6642-6646, doi:10.1073/pnas.88.15.6642 (1991).

Marshall, R. P. et al. Angiotensin II and the fibroproliferative response to acute lung injury. American journal of physiology. Lung cellular and molecular physiology 286, L156-164, doi:10.1152/ajplung.00313.2002 (2004).

Piguet, P. F., Ribaux, C., Karpuz, V., Grau, G. E. \& Kapanci, Y. Expression and localization of tumor necrosis factor-alpha and its mRNA in idiopathic pulmonary fibrosis. The American journal of pathology 143, 651-655 (1993).

Liu, J. Y. et al. Transforming growth factor-beta(1) overexpression in tumor necrosis factor-alpha receptor knockout mice induces fibroproliferative lung disease. Am J Respir Cell Mol Biol 25, 3-7, doi:10.1165/ajrcmb.25.1.4481 (2001).

growth factor in IPF. European Respiratory Journal 17, 1220 (2001). Pan, L. H., Ohtani, H., Yamauchi, K. \& Nagura, H. Co-expression of TNF alpha and IL-1 beta in human acute pulmonary fibrotic diseases: an immunohistochemical analysis. Pathology international 46, 91-99, doi:10.1111/j.1440-1827.1996.tb03584.x (1996).

Vaillant, P., Menard, O., Vignaud, J. M., Martinet, N. \& Martinet, Y. The role of cytokines in human lung fibrosis. Monaldi archives for chest disease = Archivio Monaldi per le malattie del torace / Fondazione clinica del lavoro, IRCCS [and] Istituto di clinica tisiologica e malattie apparato respiratorio, Universita di Napoli, Secondo ateneo 51, 145-152 (1996).

Martinet, Y., Rom, W. N., Grotendorst, G. R., Martin, G. R. \& Crystal, R. G. Exaggerated Spontaneous Release of Platelet-Derived Growth Factor by Alveolar Macrophages from Patients with Idiopathic Pulmonary Fibrosis. New England Journal of Medicine 317, 202-209, doi:10.1056/NEJM198707233170404 (1987).

Zhuo, Y., Zhang, J., Laboy, M. \& Lasky, J. A. Modulation of PDGF-C and PDGF-D expression during bleomycin-induced lung fibrosis. American journal of physiology. Lung cellular and molecular physiology 286, L182-188, doi:10.1152/ajplung.00083.2003 (2004).

Nakagami, H. et al. Mitogenic and antiapoptotic actions of hepatocyte growth factor through ERK, STAT3, and AKT in endothelial cells. Hypertension 37, 581-586, doi:10.1161/01.hyp.37.2.581 (2001).

Oncogene 24, 1749-1766, doi:10.1038/sj.onc.1208327 (2005).

47 Moumen, A. et al. Met signals hepatocyte survival by preventing Fas-triggered FLIP degradation in a PI3k-Akt-dependent manner. Hepatology 45, 1210-1217, doi:https://doi.org/10.1002/hep.21604 (2007). 
Matsumoto, K., Okazaki, H. \& Nakamura, T. Up-regulation of hepatocyte growth factor gene expression by interleukin-1 in human skin fibroblasts. Biochemical and biophysical research communications $\mathbf{1 8 8 ,}$ 235-243, doi:10.1016/0006-291x(92)92375-8 (1992).

49 Skibinski, G., Skibinska, A. \& James, K. The role of hepatocyte growth factor and its receptor c-met in interactions between lymphocytes and stromal cells in secondary human lymphoid organs. Immunology 102, 506-514, doi:10.1046/j.1365-2567.2001.01186.x (2001).

641

642

643

644

645

646

647

648

649

650

651

652

653

654

50 Matsumoto, K., Tajima, H., Okazaki, H. \& Nakamura, T. Negative regulation of hepatocyte growth factor gene expression in human lung fibroblasts and leukemic cells by transforming growth factor-beta 1 and glucocorticoids. J Biol Chem 267, 24917-24920 (1992).

51 Galimi, F. et al. Hepatocyte Growth Factor Is a Regulator of Monocyte-Macrophage Function. The Journal of Immunology 166, 1241, doi:10.4049/jimmunol.166.2.1241 (2001).

52 van der Voort, R. et al. Paracrine regulation of germinal center B cell adhesion through the c-methepatocyte growth factor/scatter factor pathway. $J$ Exp Med 185, 2121-2131, doi:10.1084/jem.185.12.2121 (1997).

53 Singhal, E., Kumar, P. \& Sen, P. A novel role for Bruton's tyrosine kinase in hepatocyte growth factormediated immunoregulation of dendritic cells. The Journal of biological chemistry 286, 32054-32063, doi:10.1074/jbc.M111.271247 (2011).

54 Coudriet, G. M., He, J., Trucco, M., Mars, W. M. \& Piganelli, J. D. Hepatocyte growth factor modulates interleukin-6 production in bone marrow derived macrophages: implications for inflammatory mediated diseases. PLoS One 5, e15384, doi:10.1371/journal.pone.0015384 (2010).

55 Rutella, S. et al. Hepatocyte growth factor favors monocyte differentiation into regulatory interleukin (IL)-10++IL-12low/neg accessory cells with dendritic-cell features. Blood 108, 218-227, doi:10.1182/blood-2005-08-3141 (2006).

56 Benkhoucha, M. et al. Hepatocyte growth factor inhibits CNS autoimmunity by inducing tolerogenic dendritic cells and CD25+Foxp3+ regulatory T cells. Proc Natl Acad Sci U S A 107, 6424-6429, doi:10.1073/pnas.0912437107 (2010).

57 Molnarfi, N., Benkhoucha, M., Juillard, C., Bjarnadóttir, K. \& Lalive, P. H. The neurotrophic hepatocyte growth factor induces protolerogenic human dendritic cells. Journal of neuroimmunology 267, 105-110, doi:https://doi.org/10.1016/j.jneuroim.2013.12.004 (2014).

58 Tjin, E. P. et al. Follicular dendritic cells catalyze hepatocyte growth factor (HGF) activation in the germinal center microenvironment by secreting the serine protease HGF activator. J Immunol 175, 28072813, doi:10.4049/jimmunol.175.5.2807 (2005).

59 Kazanietz, M. G., Durando, M. \& Cooke, M. CXCL13 and Its Receptor CXCR5 in Cancer: Inflammation, Immune Response, and Beyond. Front Endocrinol (Lausanne) 10, 471-471, doi:10.3389/fendo.2019.00471 (2019).

60 DePianto, D. J. et al. Heterogeneous gene expression signatures correspond to distinct lung pathologies and biomarkers of disease severity in idiopathic pulmonary fibrosis. Thorax 70, 48-56, doi:10.1136/thoraxjnl-2013-204596 (2015).

61 Vuga, L. J. et al. C-X-C motif chemokine 13 (CXCL13) is a prognostic biomarker of idiopathic pulmonary fibrosis. Am J Respir Crit Care Med 189, 966-974, doi:10.1164/rccm.201309-1592OC (2014).

62 Xue, M., Guo, Z., Zhou, L., Sun, B. \& Wang, H. in A67. STRUCTURE-FUNCTION RELATIONSHIPS American Thoracic Society International Conference Abstracts A2374-A2374 (American Thoracic Society, 2020).

63 Amelio, P. et al. Mixed Th1 and Th2 Mycobacterium tuberculosis-specific CD4 T cell responses in patients with active pulmonary tuberculosis from Tanzania. PLoS Negl Trop Dis 11, e0005817, doi:10.1371/journal.pntd.0005817 (2017). 
Table 1. Performance (area under the receiver-operating curve (AUC), sensitivity, specificity, positive and negative predictive values and likelihood ratio) of each candidate markers dichotomized into lower than, higher or equal to the cutpoint distinguishing ICU and nonICU COVID-19 patients of the discovery cohort.

\begin{tabular}{|c|c|c|c|c|c|c|c|}
\hline Marker & Cutpoint & AUC & Sensitivity & Specificity & $\begin{array}{c}\text { Positive predictive } \\
\text { value }\end{array}$ & $\begin{array}{c}\text { Negative predictive } \\
\text { value }\end{array}$ & Likelihood ratio \\
\hline HGF & 593.1 & $0.911(0.854-0.969)$ & $88.6(75.4-96.2)$ & $81.5(68.6-90.7)$ & $79.6(65.7-89.8)$ & $98.9(98.6$ - 99.2) & $4.79(2.71-8.46)$ \\
\hline CXCL13 & 119.7 & $0.875(0.801-0.948)$ & $88.6(75.4-96.2)$ & $79.6(66.5-89.4)$ & $78.0(64.0-88.5)$ & $89.6(77.3-96.5)$ & $4.35(2.54-7.45)$ \\
\hline CXCL9 & 19 & $0.869(0.801-0.936)$ & $84.1(69.9-93.4)$ & $74.1(60.3-85.0)$ & $72.5(58.3-84.1)$ & $85.1(71.7-93.8)$ & $3.24(2.03-5.18)$ \\
\hline IL-6 & 25.6 & $0.796(0.708-0.884)$ & $77.3(62.2-88.5)$ & $79.6(66.5-89.4)$ & $75.6(60.5-87.1)$ & $81.1(68.0$ - 90.6) & $3.79(2.19-6.58)$ \\
\hline CCL2 & 121.3 & $0.775(0.676-0.873)$ & $65.9(50.1-79.5)$ & $85.2(72.9-93.4)$ & $78.4(61.8-90.2)$ & $75.4(62.7-85.5)$ & $4.45(2.27-8.73)$ \\
\hline CXCL10 & 156 & $0.743(0.640-0.846)$ & $68.2(52.4-81.4)$ & $75.9(62.4-86.5)$ & $69.8(53.9-82.8)$ & $74.5(61.0-85.3)$ & $2.83(1.69-4.74)$ \\
\hline IL-1RA & 2741.7 & $0.734(0.635-0.833)$ & $79.5(64.7-90.2)$ & $61.1(46.9-74.1)$ & $62.5(48.5-75.1)$ & $78.6(63.2-89.7)$ & $2.05(1.42-2.95)$ \\
\hline CCL4 & 40 & $0.714(0.611-0.818)$ & $79.5(64.7-90.2)$ & $59.3(45.0-72.4)$ & $61.4(47.6-74.0)$ & $78.0(62.4-89.4)$ & $1.95(1.37-2.78)$ \\
\hline VEGF-A & 677.4 & $0.702(0.597-0.807)$ & $61.4(45.5$ - 75.6) & $77.8(64.4-88.0)$ & $69.2(52.4-83.0)$ & $71.2(57.9-82.2)$ & $2.76(1.59-4.79)$ \\
\hline IL-15 & 16.2 & $0.689(0.581-0.796)$ & $65.9(50.1-79.5)$ & $72.2(58.4-83.5)$ & $65.9(50.1-79.5)$ & $72.2(58.4-83.5)$ & $2.37(1.47-3.83)$ \\
\hline IL-10 & 3.1 & $0.687(0.599-0.775)$ & $50.0(34.6-65.4)$ & $88.9(77.4-95.8)$ & $78.6(59.0$ - 91.7) & $68.6(56.4-79.1)$ & $4.50(2.00-10.1)$ \\
\hline IL-1 $\beta$ & 4.325 & $0.690(0.582-0.797)$ & $69.8(53.9-82.8)$ & $70.9(57.1-82.4)$ & $65.2(49.8-78.6)$ & $75(61.1-86.0)$ & $2.4(1.5-3.8)$ \\
\hline LIF & 15.23 & $0.703(0.597-0.809)$ & $65.1(49.1-79.0)$ & $72.7(59.0-83.9)$ & $65.1(49.1-79.0)$ & $72.7(59.0$ - 83.9) & $2.4(1.5-3.9)$ \\
\hline
\end{tabular}


Table 2. Performance (AUC, sensitivity, specificity, positive and negative predictive values and likelihood ratio) of each candidate markers dichotomized into lower than, higher or equal to the cutpoint distinguishing ICU and non-ICU COVID-19 patients in FCS and LUH-2 validation cohorts.

\begin{tabular}{|c|c|c|c|c|c|c|c|}
\hline Marker & Cutpoint & AUC & Sensitivity & Specificity & Positive predictive value & Negative predictive value & Likelihood ratio \\
\hline \multicolumn{8}{|c|}{ FCS validation cohort } \\
\hline HGF & 593.1 & $0.976(0.948-1.000)$ & $87.1(70.2-96.4)$ & $93.5(78.6-99.2)$ & $93.1(77.2-99.2)$ & $87.9(71.8-96.6)$ & $13.5(3.5-51.9)$ \\
\hline CXCL13 & 119.7 & $0.903(0.832-0.974)$ & $96.8(83.3-99.9)$ & $58.1(39.1-75.5)$ & $69.8(53.9-82.8)$ & $94.7(74.0-99.9)$ & $2.3(1.5-3.5)$ \\
\hline CXCL9 & 19.0 & $0.814(0.706-0.921)$ & $51.6(33.1-69.8)$ & $87.1(70.2-96.4)$ & $80.0(56.3-94.3)$ & $64.3(48.0-78.4)$ & $4.0(1.5-10.6)$ \\
\hline IL-6 & 25.6 & $0.661(0.578-0.745)$ & $16.1(5.5-33.7)$ & $100(88.8-100)$ & $100(47.8-100)$ & $54.4(40.7-67.6)$ & Not computable \\
\hline CCL2 & 121.3 & $0.745(0.622-0.867)$ & $29.0(14.2-48.0)$ & $100(88.8-100)$ & $100(66.4-100)$ & $58.5(44.1-71.9)$ & Not computable \\
\hline CXCL10 & 156.0 & $0.782(0.663-0.901)$ & $25.8(11.9-44.6)$ & $93.5(78.6-99.2)$ & $80.0(44.4-97.5)$ & $55.8(41.3-69.5)$ & $4.0(0.9-17.4)$ \\
\hline IL-1RA & 2741.7 & $0.803(0.692-0.915)$ & $35.5(19.2-54.6)$ & $96.8(83.3-99.9)$ & $91.7(61.5-99.8)$ & $60.0(45.2-73.6)$ & $11.0(1.5-80.1)$ \\
\hline CCL4 & 40.0 & $0.714(0.581-0.846)$ & $100(88.8-100)$ & $9.7(2.0-25.8)$ & $52.5(39.1-65.7)$ & $100(29.2-100)$ & $1.1(1.0-1.2)$ \\
\hline VEGF-A & 677.4 & $0.856(0.765-0.947)$ & $83.9(66.3-94.5)$ & $58.1(39.1-75.5)$ & $66.7(49.8-80.9)$ & $78.3(56.3-92.5)$ & $2.0(1.3-3.1)$ \\
\hline IL-15 & 16.2 & $0.766(0.647-0.885)$ & $38.7(21.8-57.8)$ & $90.3(74.2-98.0)$ & $80.0(51.9-95.7)$ & $59.6(44.3-73.6)$ & $4.0(1.3-12.8)$ \\
\hline IL-10 & 3.1 & $0.594(0.512-0.676)$ & $22.6(9.6-41.1)$ & $96.8(83.3-99.9)$ & $87.5(47.3-99.7)$ & $55.6(41.4-69.1)$ & $7.0(0.9-53.6)$ \\
\hline IL-1 $\beta$ & 4.325 & $0.604(0.473-0.734)$ & $38.7(21.8-57.8)$ & $77.4(58.9-90.4)$ & $63.2(38.4-83.7)$ & $55.8(39.9-70.9)$ & $1.7(0.8-3.8)$ \\
\hline LIF & 15.23 & $0.652(0.540-0.765)$ & $16.1(5.5-33.7)$ & $96.8(83.3-99.9)$ & $83.3(35.9-99.6)$ & $53.6(39.7-67.0)$ & $5.0(0.6-40.4)$ \\
\hline \multicolumn{8}{|c|}{ LUH-2 validation cohort } \\
\hline HGF & 593.1 & $0.976(0.948-1.000)$ & $87.1(70.2-96.4)$ & $93.5(78.6-99.2)$ & $93.1(77.2-99.2)$ & $87.9(71.8-96.6)$ & $13.5(3.5-51.9)$ \\
\hline CXCL13 & 119.7 & $0.903(0.832-0.974)$ & $96.8(83.3-99.9)$ & $58.1(39.1-75.5)$ & $69.8(53.9-82.8)$ & $94.7(74.0$ - 99.9) & $2.3(1.5-3.5)$ \\
\hline CXCL9 & 19.0 & $0.814(0.706-0.921)$ & $51.6(33.1-69.8)$ & $87.1(70.2-96.4)$ & $80.0(56.3-94.3)$ & $64.3(48.0-78.4)$ & $4.0(1.5-10.6)$ \\
\hline IL-6 & 25.6 & $0.661(0.578-0.745)$ & $16.1(5.5-33.7)$ & $100(88.8-100)$ & $100(47.8-100)$ & $54.4(40.7-67.6)$ & Not computable \\
\hline CCL2 & 121.3 & $0.745(0.622-0.867)$ & $29.0(14.2-48.0)$ & $100(88.8-100)$ & $100(66.4-100)$ & $58.5(44.1-71.9)$ & Not computable \\
\hline CXCL10 & 156.0 & $0.782(0.663-0.901)$ & $25.8(11.9-44.6)$ & $93.5(78.6-99.2)$ & $80.0(44.4-97.5)$ & $55.8(41.3-69.5)$ & $4.0(0.9-17.4)$ \\
\hline IL-1RA & 2741.7 & $0.803(0.692-0.915)$ & $35.5(19.2-54.6)$ & $96.8(83.3-99.9)$ & $91.7(61.5-99.8)$ & $60.0(45.2-73.6)$ & $11.0(1.5-80.1)$ \\
\hline CCL4 & 40.0 & $0.714(0.581-0.846)$ & $100(88.8-100)$ & $9.7(2.0-25.8)$ & $52.5(39.1-65.7)$ & $100(29.2-100)$ & $1.1(1.0-1.2)$ \\
\hline VEGF-A & 677.4 & $0.856(0.765-0.947)$ & $83.9(66.3-94.5)$ & $58.1(39.1-75.5)$ & $66.7(49.8-80.9)$ & $78.3(56.3-92.5)$ & $2.0(1.3-3.1)$ \\
\hline IL-15 & 16.2 & $0.766(0.647-0.885)$ & $38.7(21.8-57.8)$ & $90.3(74.2-98.0)$ & $80.0(51.9-95.7)$ & $59.6(44.3-73.6)$ & $4.0(1.3-12.8)$ \\
\hline IL-10 & 3.1 & $0.594(0.512-0.676)$ & $22.6(9.6-41.1)$ & $96.8(83.3-99.9)$ & $87.5(47.3-99.7)$ & $55.6(41.4-69.1)$ & $7.0(0.9-53.6)$ \\
\hline
\end{tabular}


Table 3. Performance (sensitivity, specificity, positive and negative predictive values and the likelihood ratio) of the combination of HGF and CXCL13 to further improve the discrimination between ICU and non-ICU COVID-19 patients.

\begin{tabular}{cccccc}
\hline Cohort & N & Sensitivity & Specificity & Positive predictive value & Negative predictive value \\
\hline LUH-1 & 98 & $\begin{array}{c}79.1(64.0- \\
90.0)\end{array}$ & $94.5(84.9-98.9)$ & $91.9(78.1-98.3)$ & $85.2(73.8-93.0)$ \\
LUH-2 & 47 & $\begin{array}{c}81.8(48.2- \\
97.7)\end{array}$ & $94.4(81.3-99.3)$ & $81.8(48.2-97.7)$ & $94.4(81.3-99.3)$ \\
FCS & 62 & $\begin{array}{c}87.1(70.2- \\
96.4)\end{array}$ & $93.5(78.6-99.2)$ & $93.1(77.2-99.2)$ & $87.9(71.8-96.6)$
\end{tabular}


Table 4. Performance of each candidate markers dichotomized into lower than or higher than the cutpoint or of the combination of HGF and CXCL13 to predict death during the follow-up of COVID-19 patients enrolled in LUH-1, LUH-2 and the FCS cohorts.

\begin{tabular}{cccccc}
\hline Marker & Low & High & p-value $*$ & Hazard ratio* $^{*}$ & p-value $\|$ \\
\hline HGF & $5(4.6)$ & $13(14.9)$ & 0.012 & $1.53(0.29-8.18)$ & 0.621 \\
CXCL13 & $2(2.4)$ & $16(14.0)$ & 0.005 & $4.94(0.85-28.6)$ & 0.075 \\
CXCL9 & $5(4.6)$ & $13(14.6)$ & 0.016 & $1.02(0.32-3.26)$ & 0.980 \\
IL-6 & $10(7.1)$ & $8(14.3)$ & 0.114 & $1.33(0.45-3.87)$ & 0.606 \\
CCL2 & $12(8.1)$ & $6(12.5)$ & 0.352 & $0.66(0.21-2.03)$ & 0.463 \\
CXCL10 & $9(6.7)$ & $9(14.5)$ & 0.076 & $3.73(1.14-12.2)$ & 0.029 \\
IL-1RA & $8(6.3)$ & $10(14.3)$ & 0.063 & $2.39(0.73-7.82)$ & 0.151 \\
CCL4 & $2(4.6)$ & $16(10.5)$ & 0.230 & $2.57(0.48-13.7)$ & 0.269 \\
VEGF-A & $8(8.0)$ & $10(10.3)$ & 0.574 & $1.23(0.40-3.74)$ & 0.721 \\
IL-15 & $11(8.7)$ & $7(9.9)$ & 0.792 & $0.85(0.28-2.58)$ & 0.780 \\
IL-10 & $13(8.5)$ & $5(11.4)$ & 0.561 & $0.81(0.26-2.50)$ & 0.712 \\
IL-1 3 & $12(10.1)$ & $6(7.7)$ & 0.569 & $0.45(0.15-1.36)$ & 0.158 \\
LIF & $12(8.1)$ & $6(12.2)$ & 0.384 & $0.74(0.24-2.26)$ & 0.597 \\
Combination of HGF and CXCL13 & & & \\
HGF/CXCL13 & $1(1.5)$ & $17(13.3)$ & 0.006 & $8.80(0.96-80.3)$ & 0.054
\end{tabular}

The first two columns indicate the percentage of subjects within a given category (low or high levels) who died during follow-up, all cohorts together. *, adjusted for age (continuous), ICU stay

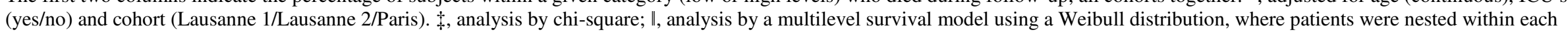
cohort. 


\section{Figure legends}

Figure 1. Distribution of CD4 T cell lineage and phosphoprotein signaling profiles in nonICU and ICU COVID-19 patients. A. Frequencies of Th1 (CXCR3+T-bet+), Th2 (CCR4+Gata-3+), Th17 (CCR6+RoR- $\gamma \mathrm{t}+$ ) and Treg (CD25+CD127-FoxP3+) CD4 T cell subpopulations in healthy subjects $(\mathrm{N}=146)$, non-ICU $(\mathrm{N}=50)$ and ICU $(\mathrm{N}=25)$ patients. B. Mean signal intensity of ex vivo phospho-STAT1 (pSTAT1), pSTAT3, pSTAT5, p38, pMAPKAP2, pNFkB, pCREB, pS6 and pERK1/2 in healthy subjects $(\mathrm{N}=39)$, non-ICU $(\mathrm{N}=33)$ and ICU $(\mathrm{N}=29)$ patients. Blue plots correspond to healthy subjects (H.S), green plots corresponds to non-ICU patients and red plots correspond to ICU patients. Black stars indicate statistical significance between ICU or non-ICU patients and healthy subjects. Statistical significance ( $P$ values) was obtained using Kruskal-Wallis test, using a Bonferroni correction. ${ }^{*}=P<0.05$; $* *=P<0.01 ; * * *=P<0.001$.

Figure 2. Serum cytokine, soluble cytokine receptor, chemokine and growth factor profiles in non-ICU and ICU COVID-19 patients. Levels of cytokines (IL-1 $\alpha$, IL-1 $\beta$, IL-6, TNF- $\alpha$, IL-18, IL-27, IL-12p70, IFN $\alpha 2$, IL-10, IL-23, IL-9, IFN $\gamma$, IL-4, IL-5, IL-13, IL-31, IL17A, IL-21, IL-22, IL-2, IL-7, IL15, BAFF, TNF- $\beta$ ) cytokine receptor (IL-1RA), chemokines (CCL2, CCL3, CCL4, CCL5, CCL11, CXCL1, CXCL8, CXCL9, CXCL10, CXCL12, CXCL13) and growth factors (NGF- $\beta$, BDNF, EGF, FGF-2, HGH, LIF, PDGF-BB, PIGF-1, SCF, VEGF-A, VEGF-D, G-CSF, GM-CSF) in healthy subjects (N=450), non-ICU $(\mathrm{N}=55)$ and ICU (N=43) patients. Blue plots correspond to healthy subjects (H.S), green plots corresponds to non-ICU patients and red plots correspond to ICU patients. Dotted line represents limit of detection. Black stars indicate statistical significance between ICU or nonICU patients and healthy subjects. Red stars indicate statistical significance between ICU and non-ICU patients. Statistical significance ( $P$ values) was obtained using Kruskal-Wallis test, using a Bonferroni correction. $*=P<0.05 ; * *=P<0.01 ; * * *=P<0.001$.

Figure 3. Individual performances of IL-10, CCL2, CCL4, CXCL13, IL-1RA, IL-6, IL15, VEGF-A, CXCL9, CXCL10 and HGF serum levels to discriminate between ICU and non-ICU COVID-19 patients enrolled in the 'discovery' cohort. Receiver-operating characteristic (ROC) curves and corresponding area under the curve (AUC) values of IL-10, CCL2, CCL4, CXCL13, IL-1RA, IL-6, IL-15, VEGF-A, CXCL9, CXCL10 and HGF discriminating ICU from non-ICU SARS-CoV2 infected individuals enrolled in the 'discovery' cohort ( $\mathrm{N}=98)$. 


\section{Acknowledgements}

We are grateful to Nathalie Felix, Stéphanie Gregoretti, Aurélie Myard, Nadine Do Rosario, Amélie Muralti, Thibaut Decaillon, Emmanuelle Medjitna, Philippe Kiehl, Gonzalo Tapia, Manuela Lavelli, Michael Moulin, Manon Geiser, Line Esteves-Leuenberger, Olivia Munoz and Riddhima Banga for technical assistance. We are grateful to Rosemary Hottinger and Fabio Candotti for study management. Finally, Aaron Weddle and John Weddle for their assistance with the figures. This work was supported by Swiss National Science Foundation Grants 320030_173071 to M. Perreau and 31CA30_196852 to G. Pantaleo.

\section{Competing interests}

The authors declare no competing interest. 
Figure 1

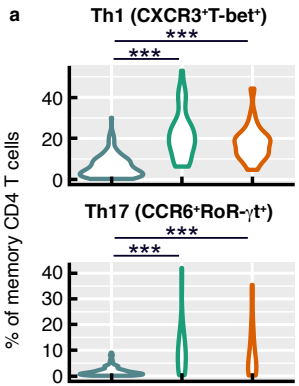

Th2 (CCR4+Gata-3+)

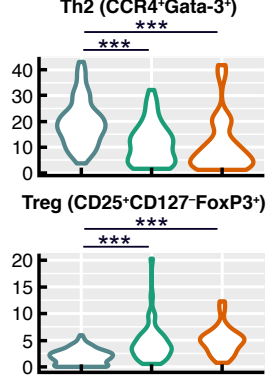

HS ( $N=146)$

non-ICU $(\mathrm{N}=50)$

ICU ( $=25)$

b
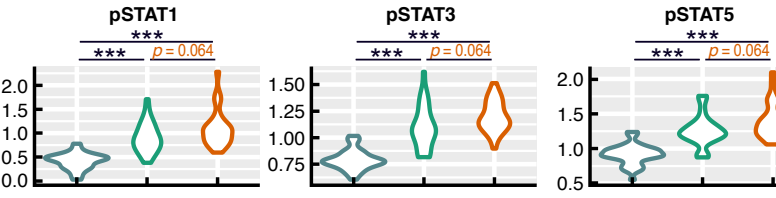

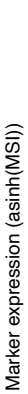
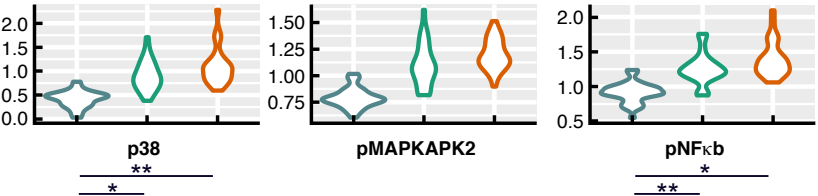

pMAPKAPK2

pNFkb
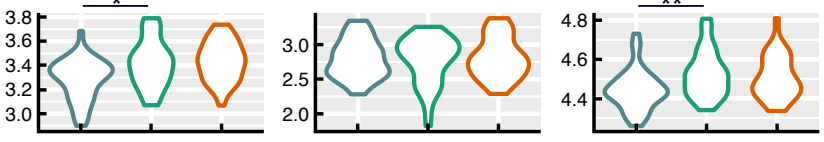

PCREB

$\frac{\star \star *}{\star * *} p=0.064$
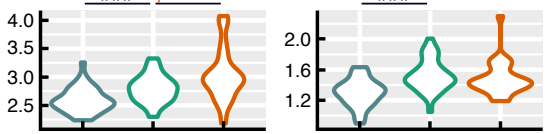

$\mathrm{HS}(\mathrm{N}=39)$

non-ICU $(\mathrm{N}=33)$
pERK1/2

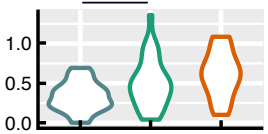

$\operatorname{ICU}(\mathrm{N}=29)$ 
Figure 2

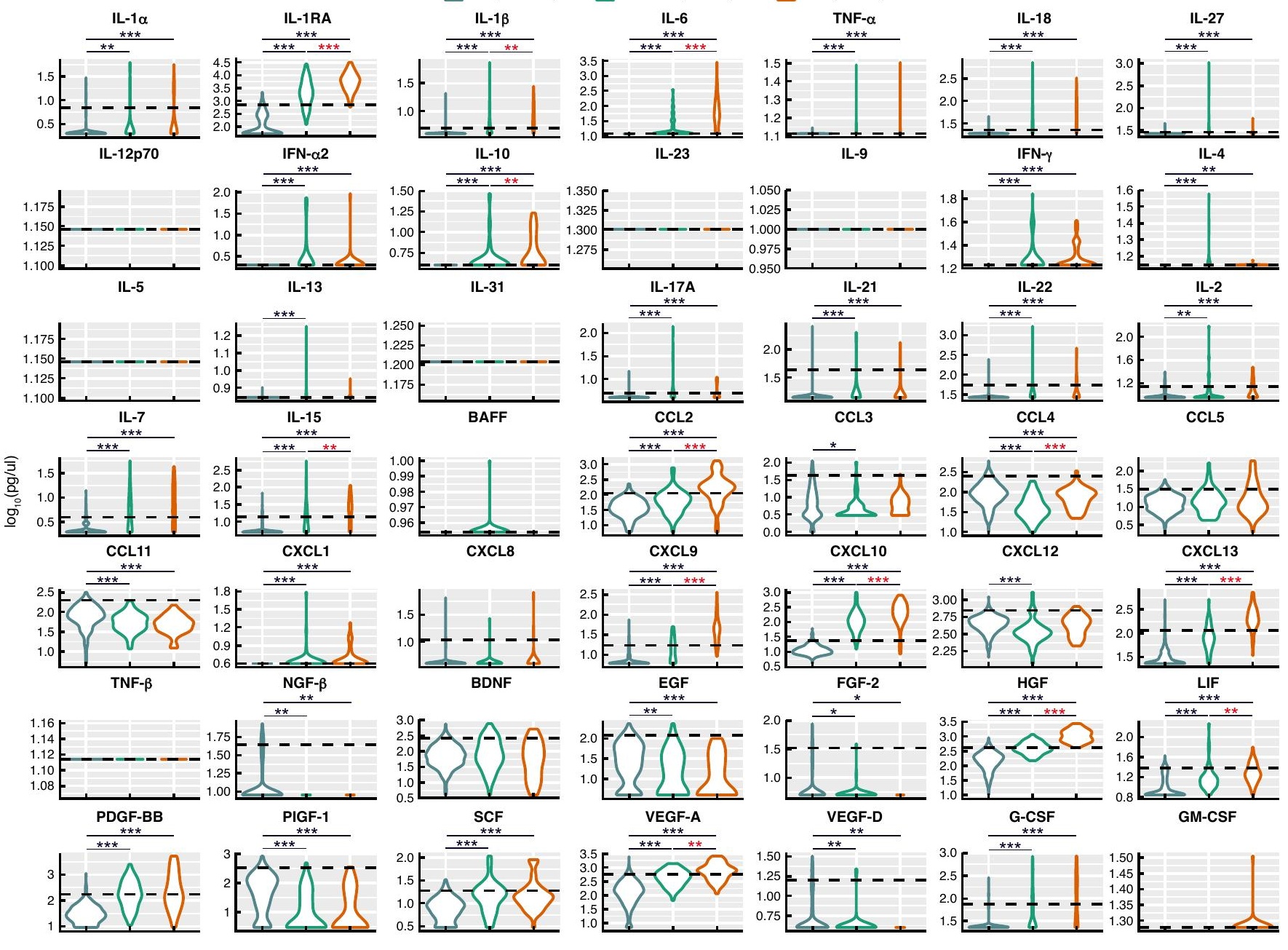




\section{Figure 3}
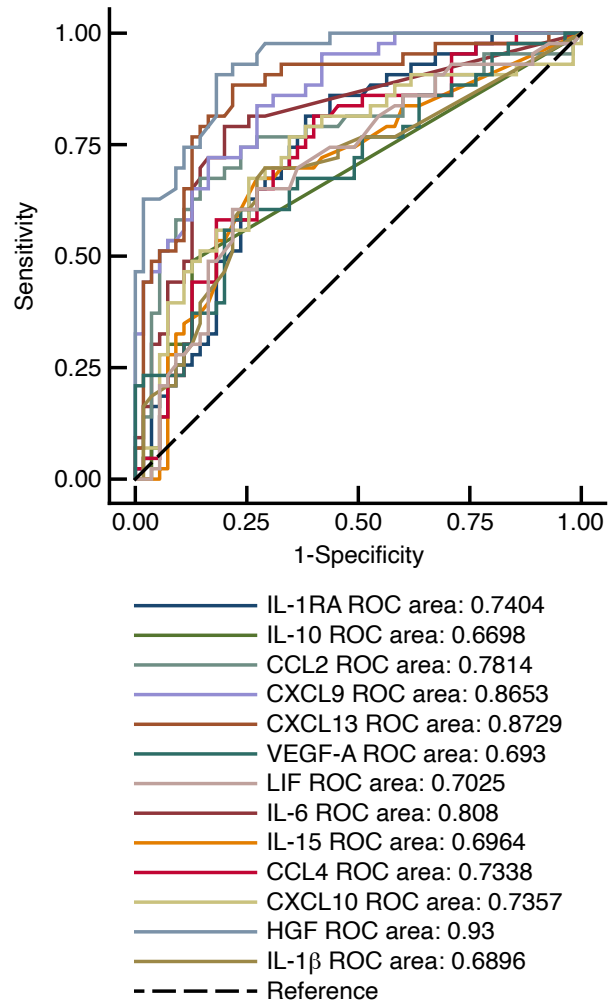
Figures
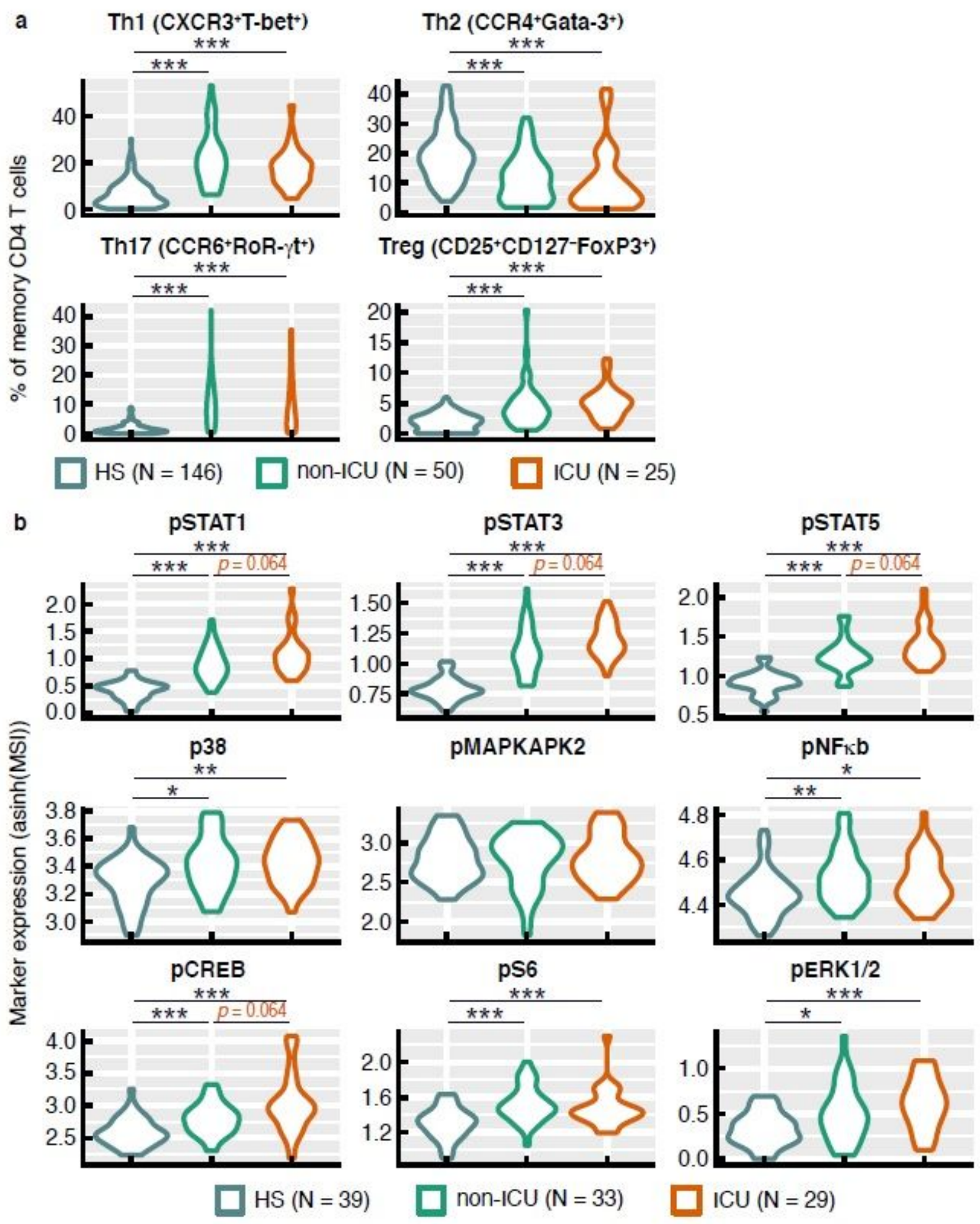

Figure 1

Distribution of CD4 T cell lineage and phosphoprotein signaling profiles in non- ICU and ICU COVID-19 patients. A. Frequencies of Th1 (CXCR3+T-bet+), Th2 (CCR4+Gata-3+), Th17 (CCR6+RoR-yt+) and Treg (CD25+CD127-FoxP3+) CD4 T cell subpopulations in healthy subjects $(N=146)$, non-ICU $(N=50)$ and ICU 
$(\mathrm{N}=25)$ patients. $B$. Mean signal intensity of ex vivo phospho-STAT1 (pSTAT1), pSTAT3, pSTAT5, p38, pMAPKAP2, pNFkB, pCREB, pS6 and pERK1/2 in healthy subjects $(\mathrm{N}=39)$, non-ICU $(\mathrm{N}=33)$ and ICU $(\mathrm{N}=29)$ patients. Blue plots correspond to healthy subjects (H.S), green plots corresponds to non-ICU patients and red plots correspond to ICU patients. Black stars indicate statistical significance between ICU or non-ICU patients and healthy subjects. Statistical significance ( $P$ values) was obtained using Kruskal-Wallis test, using a Bonferroni correction. ${ }^{*}=\mathrm{P}<0.05 ; * *=\mathrm{P}<0.01 ; * * *=\mathrm{P}<0.001$.

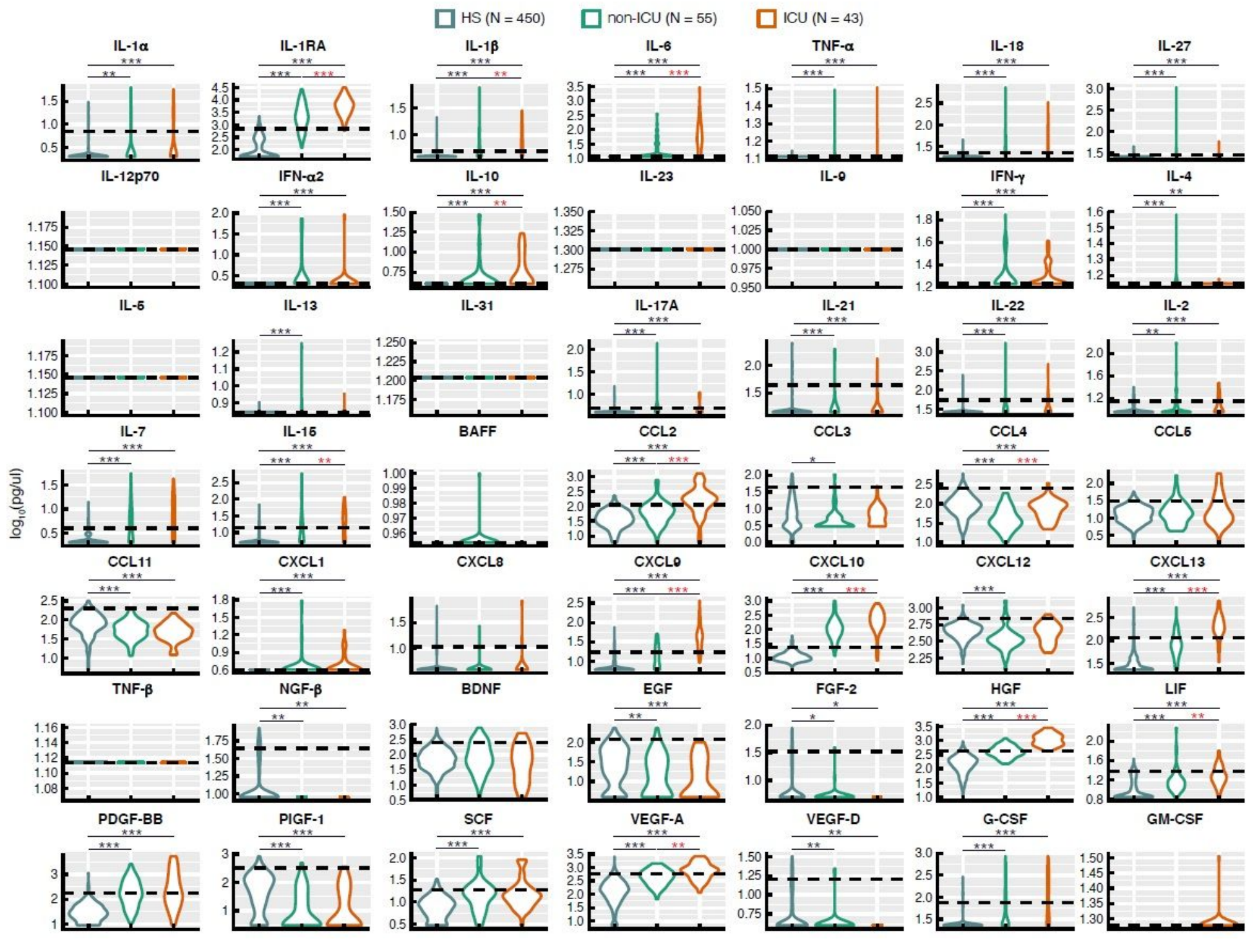

Figure 2

Serum cytokine, soluble cytokine receptor, chemokine and growth factor profiles in non-ICU and ICU COVID-19 patients. Levels of cytokines (IL-1a, IL-1 $\beta$, IL-6, TNF-a, IL-18, IL-27, IL-12p70, IFNa2, IL-10, IL-23, IL-9, IFNy, IL-4, IL-5, IL-13, IL-31, IL- 17A, IL-21, IL-22, IL-2, IL-7, IL15, BAFF, TNF- $\beta$ ) cytokine receptor (IL1RA), chemokines (CCL2, CCL3, CCL4, CCL5, CCL11, CXCL1, CXCL8, CXCL9, CXCL10, CXCL12, CXCL13) and growth factors (NGF- $\beta$, BDNF, EGF, FGF-2, HGH, LIF, PDGF-BB, PIGF-1, SCF, VEGF-A, VEGF-D, G-CSF, GM-CSF) in healthy subjects $(\mathrm{N}=450)$, non-ICU $(\mathrm{N}=55)$ and ICU $(\mathrm{N}=43)$ patients. Blue plots correspond to healthy subjects (H.S), green plots corresponds to non-ICU patients and red plots correspond to ICU 
patients. Dotted line represents limit of detection. Black stars indicate statistical significance between ICU or non- ICU patients and healthy subjects. Red stars indicate statistical significance between ICU and nonICU patients. Statistical significance (P values) was obtained using Kruskal-Wallis test, using a Bonferroni correction. ${ }^{*}=\mathrm{P}<0.05 ; * \star=P<0.01 ; * * *=P<0.001$.
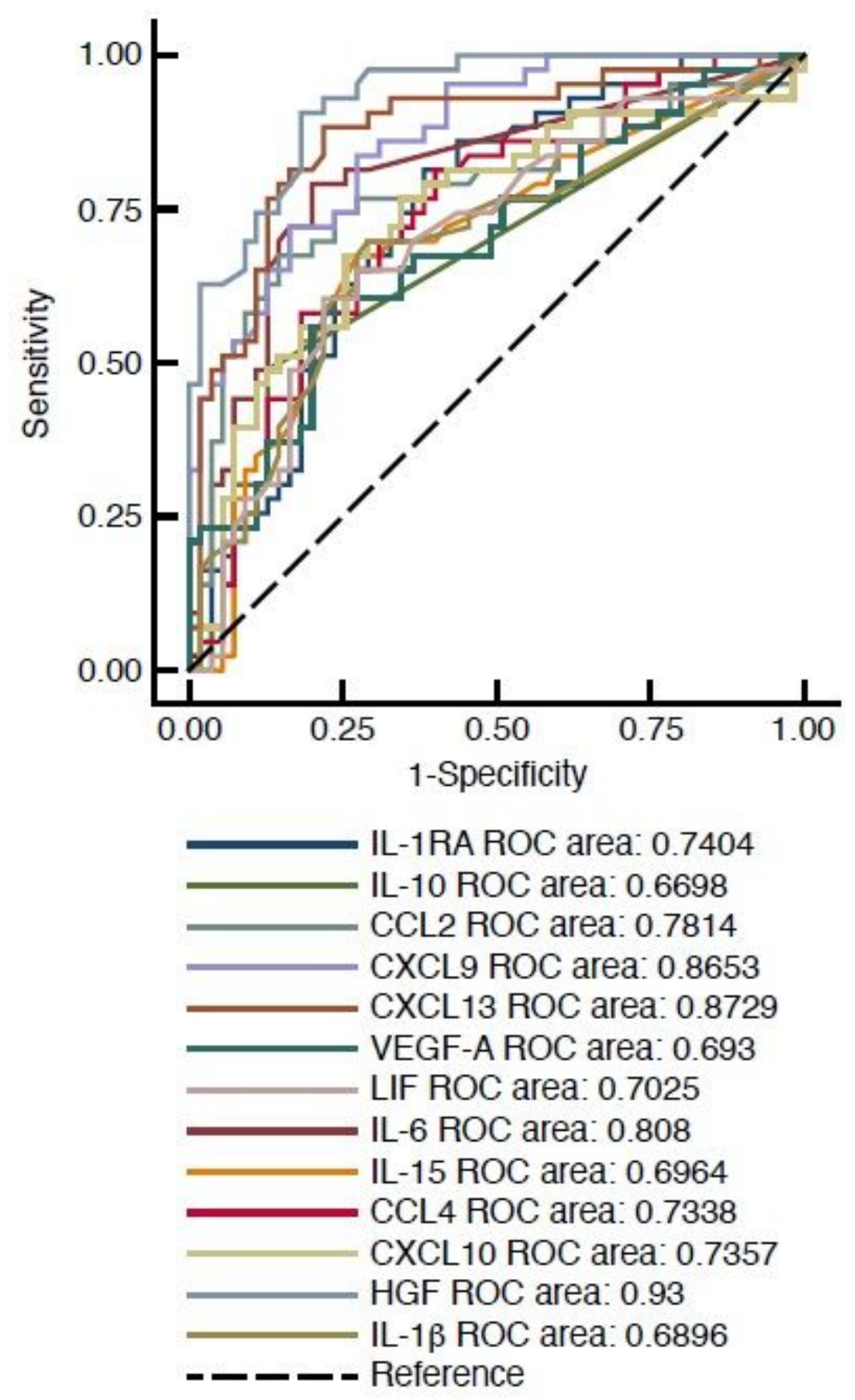

Figure 3

Individual performances of IL-10, CCL2, CCL4, CXCL13, IL-1RA, IL-6, IL-15, VEGF-A, CXCL9, CXCL10 and HGF serum levels to discriminate between ICU and non-ICU COVID-19 patients enrolled in the 'discovery' cohort. Receiver-operating characteristic (ROC) curves and corresponding area under the curve (AUC) 
values of IL-10, CCL2, CCL4, CXCL13, IL-1RA, IL-6, IL-15, VEGF-A, CXCL9, CXCL10 and HGF discriminating ICU from non-ICU SARS-CoV2 infected individuals enrolled in the 'discovery' cohort $(\mathrm{N}=98)$.

\section{Supplementary Files}

This is a list of supplementary files associated with this preprint. Click to download.

- Supplementaldata1422021.pdf 